\title{
Influence of Heat Generation/Absorption and Stagnation point on Polystyrene -TiO2/H 20 Hybrid Nanofluid
}

Sadaf Masood ( $\nabla$ maliksadafirfan12@gmail.com )

Riphah International University, Islamabad

Muhammad Farooq

University of Haripur

\section{Research Article}

Keywords: Hybrid Nanofluid, Stagnation point, Stretching sheet, Heat generation/absorption, Entropy generation

Posted Date: March 11th, 2021

DOI: https://doi.org/10.21203/rs.3.rs-271684/v1

License: (c) (i) This work is licensed under a Creative Commons Attribution 4.0 International License.

Read Full License 


\title{
Influence of Heat Generation/Absorption and Stagnation point on Polystyrene $-\mathrm{TiO}_{2} / \mathrm{H}_{2} \mathrm{O}$ Hybrid Nanofluid
}

\author{
${\text { Sadaf } \operatorname{Masood}^{a} \text {, M. Farooq }}^{b}$ \\ ${ }^{a}$ Department of Mathematics, Riphah International University, Islamabad 44000, Pakistan \\ ${ }^{b}$ Department of Pure and Applied Mathematics, University of Haripur, Haripur, KPK, Pakistan
}

Abstract: This article focuses on hybrid nanofluid flow induced by stretched surface. The present context covers stagnation point flow of a hybrid nanofluid with the effect of heat generation/absorption. Currently most famous class of nanofluids is Hybrid nanofluid. It contains polystyrene and titanium oxide as a nanoparticles and water as a base fluid. First time attributes of heat transfer are evaluated by utilizing polystyrene- $\mathrm{TiO}_{2} / \mathrm{H}_{2} \mathrm{O}$ hybrid nanofluid with heat generation/absorption. Partial differential equations are converted into ordinary differential equation by using appropriate transformations for heat and velocity. Homotopy analysis method is operated for solution of ordinary differential equations. Flow and heat are disclosed graphically for unlike parameters. Resistive force and heat transfer rate is deliberated mathematically and graphically. It is deduced that velocity field enhanced for velocity ratio parameter whereas temperature field grows for heat generation/absorption coefficient. To judge the production of any engineering system entropy generation is also calculated. It is noticed that entropy generation grows for Prandtl number and Eckert number while it shows opposite behavior for temperature difference parameter.

Key-words: Hybrid Nanofluid; Stagnation point; Stretching sheet; Heat generation/absorption; Entropy generation.

\section{Introduction}

Heat transmission plays a vital role in many respects for instance in refrigeration, power generation, thermoelectric devices, heat exchangers, roofing materials, food processing, radiative cooling and thermal energy storage etc. Therefore it is advantageous to enhance the production of heat transfer machines adopted in these areas. Thermal conductivity is the crucial framework in heat transfer problems. Ethylene glycol, water and oils have low thermal conductivity. Nanomaterials like oxides of metals, carbides etc are included in the host fluid for intensification of thermal conductivity. Choi [1] instigated about nanofluids. Hybrid nanofluids seeks the intention of researchers and scientists currently. It consists of two or more non-identical particles having size less than $100 \mathrm{~nm}$. Here, we take polystyrene and titanium oxide as nanoparticles due to their wide use in pharmaceuticals, automtive industary, IT equipments(TV, Computers, laptops), food packing industary, construction, household industary, cosmetics, fabrics and textiles. Waini et al. [2] avails bvp4c to conclude the heat transfer of hybrid nanofluid with shear flow. They checked the stability of a solutions and concluded that one solution is stable from the dual solutions. Influence of CNT - Fe $3 \mathrm{O}_{4} / \mathrm{H}_{2} \mathrm{O}$ hybrid nanofluid on infinite rotating disk was studied by Tassaddiq et al . [3]. They observed that heat trasfer of CNT - $\mathrm{Fe}_{3} \mathrm{O}_{4} / \mathrm{H} \quad 2 \mathrm{O}$ hybrid nanofluid is greater as compared with $\mathrm{Fe} \quad 3 \quad \mathrm{O}_{4} / \mathrm{H}$ 2 O. Tayebi et al. [4] analyzed the attributes of hybrid nanofluid (containg $\mathrm{Cu}$ and $\mathrm{Al} 2 \mathrm{O} 3$ 
nanoparticles) bounded by elliptical cylinders with natural convection. He investigated that entropy generation grows for highter Rayleigh number. Peristaltic flow of hybrid nanofluid with entropy generation was executed by Zahid et al. [5]. Shooting method is utilized to perceive numerical solutions. It is noticed that enhanced Hall parameter decays the heat transfer rate as well as entropy generation. Yusuf et al.[6] takes stretching sheet with nonlinear radiations on hybrid nanofluid and investigated that entropy generation rate rises for radiation parameter. Wanatasanapan et al. [7] takes temperature range $30-70^{\circ} \mathrm{C}$ and fixed volume fraction $1.0 \%$ of $\mathrm{TiO} 2$ and $\mathrm{A} 12 \mathrm{O} 3$ particles. They concluded that hybrid nanofluid with 50:50 ratio at $70{ }^{0} \mathrm{C}$ has maximum thermal conductivity. Matlab was used by Said et al. [8] for hybrid nanofluid at distinct concentrations. Enhanced nanoparticle volume concentration grows the entropy generation. $19.14 \%$ thermal conductivity enhancement was noticed at $60{ }^{0} C$. Abbas at al. [9] considered a hybrid nanofluid in a moving cylinder with slip and inclined MHD. Consequences of silver-CuO/H $2 \mathrm{O}$ nanofluid with stagnation point and stretching sheet are discussed by Arani et al. [10]. They used R-K method with shooting technique. They conclude that heat transfer rate grows $100 \%$ for hybrid nanofluid with suction/injection parameter. Enhancement of heat transfer in a car radiator with hybrid nanofluid was studied by Li et al. [11]. He found that at $0.4 \%$ volume fraction $32.01 \%$ thermal conductivity rises. Outcomes of hybrid nanofluid over exponentially stretching sheet with heat generation was examined by Zainal et al. [12]. Suction parameter strengthens the rate of heat transfer.

Excessive applications of stagnation point flow of Newtonian as well as Non Newtonian fluid seeks the researchers intention. A point where fluid is static by the object is called stagnation point.It is classified as oblique and orthogonal stagnation point. Stagnation point undergoes a highest pressure and highest heat transmission. Innumerable applications of stagnation point flows in engineering, home industary, aerodynamic industary and in metallurgy are noticed. They include cooling of plates, nuclear reactor cooling, tinning of wires and wire drawing etc. Naganthran et al. [13] described the flow of viscoelastic fluid past a shrinking sheet with oblique stagnation point. They concluded that enhanced mass flux parameter strengthens the heat transfer rate. Consequences of chemical reaction on CNTs along with stagnation point was founded by Khan et al. [14]. Magnified velocity ratio parameter decays the drag force. Ascendancy of Maxwell fluid with suction/injection was illustrated by Ahmed et al. [15]. Porous rotating disk was also considered. Numerical results showed that rotation parameter enhanced the nusselt number at the surface. Moshkin et al. [16] elaborated the flow of unsteady Maxwell fluid by transforming the equations into the Lagrangian coordibates. Weidman [17] contemplated a flow along a rotating plate and revealed the influence of Hiemenz stagnation point on this plate. He gave good comparision with Hannah's consideration. The out turn of Homann stagnation point on a non Newtonian fluid regarding a stable plate was initiated by Mahapatra et al. [18]. They deduced that viscoelastic parameter grows the velocity profile. Azhar et al. [19] studied about heat generation and viscous dissipation of Jeffrey fluid along with stagnation point. Stretching ratio parameter enhanced the drag force as well as Sherwood number. Flow of MHD Carreau fluid induced by stretching surface was instigated by Chu et al. [20]. He examined flow about stagnation point. Both heat and mass will transfer more fastly by 
increasing velocity ratio parameter. Shah et al [21] takes a Riga plate and found the out turns of stagnation point and mixed convection along with porous medium. Darcy number decays the velocity field while grows the skin friction. Awan et al. [22] assumed MHD second grade fluid with oblique stagnation point. The flow was induced by oscillatory surface. He showed that Sherwood number and heat transfer rate boosts up for larger suction parameter.

Conception of entropy in thermodynamic system was prescribed by Rudolf Clausius in 1850s. It is the amount of thermal energy per unit temperature which is unattainable for performing beneficial tasks. The quantity of entropy assembled in irreversible processes is termed as entropy production. For example heat exchange, heat engines, fluid flows, heat pumps, power plants, air conditioners and refrigeneration etc. It determines the execution of thermodynamical system. At first Bejan [23] studied about entropy generation. He explained the significant steps of entropy depreciation. Gholamalipour et al. [24] studied the entropy generation of nanofluid in a permeable annulus. For lesser Darcy and Rayleigh number greater disturbance in entropy producution is noticeable. Dutta et al. [25] considered a rhombic shape closed pattern pervaded by $\mathrm{Cu}$-water nanofluid and investigates about entropy generation. He showed that increment in Ha decays the entropy production rate. Khan et al. [26] discussed the impact of joule heating on casson fluid passing through a revolving cylinder. Entropy generation shows increasing trend for larger Brinkman number. Attributes of entropy production in Newtonian fluid with Darcy model was analyzed by Ambreen et al. [27]. Cho [28] takes a square cavity whose some walls are heated and filled it by $\mathrm{Cu}$-water nanofluid. Along this he considered a porous medium inside the cavity and then measure the entropy production rate. For a fixed Rayleigh number, entropy rate enhanced with enlarged Darcy number. Influence of natural convection in elliptical cavity pervaded by hybrid nanofluid was inspected by Tayebi et al. [29]. Zahid et al. [30] explained that low entropy production occurs for higher Hall parameter. Li et al. [31] examined the thermal radiation effect in a tilted square cavity. He also analyzed the entropy production rate here and found that Rayleig number grows the entropy production rate. Sachica et al. [32] scrutinized the $\mathrm{Al}_{2} \mathrm{O}_{3}$-water nanofluid in a rectangular channel and numerically investigate it. Nano particle volume fraction decreases the entropy generation rate.

Extraordinary enhancement in thermal conductivity is noticed for hybrid nanofluids in comparision with ordinary nanofluids. Therefore have innumerable applications in home industary, automative industary, engineering, for cancer treatment, cosmetics, pharmaceuticals, food pakaging, papaer plastics, fabrics, ceramics, paints, food colorants and in soaps as well . Here the key objective is to discuss the characteristics of polystyrene- $\mathrm{TiO}_{2} / \mathrm{H}_{2} \mathrm{O}$ hybrid nanofluid flow with heat generation/absorption. Stagnation point is also contemplated in momentum equation. We take advantage of congrous transformations for transmutation of partial differential equations into nondimensionalized ordinary differential equations. Homotopic methodology [33-39] is executed for series solution. Ramification of incompatible parameters are interpreted graphically. Mathematical expression of drag force is calculated and nusselt number is manifested graphically. Entropy generation rate is also exposed through graphs.

\section{Formulation}

Analysis of two dimensional hybrid nanofluid suppressed with polystyrene and titanium oxide $\left(\mathrm{TiO}_{2}\right)$ particles has carried out.Influence of stagnation point on flow pattern is also discussed. Impact of heat generation/absorption is also figure out. We take stretching velocity 
$u=U_{w}(x)=a x$ at $y=0$. Free stream velocity $u=U_{e}(x)=b x$ is considered at $y \rightarrow \infty$. Persistent temperature is presumed at both plate surface and ambient fluid.

After implementation of boundary layer approximation the ruling equations appears as:

$\frac{\partial u}{\partial x}+\frac{\partial v}{\partial y}=0$

$u \frac{\partial u}{\partial x}+v \frac{\partial u}{\partial y}=U_{e} \frac{d U_{e}}{d x}+v_{h n f} \frac{\partial^{2} u}{\partial y^{2}}$

$u \frac{\partial T}{\partial x}+v \frac{\partial T}{\partial y}=\alpha_{h n f} \frac{\partial^{2} T}{\partial y^{2}}+\frac{Q}{\left(\rho C_{p}\right)_{h n f}}$,

Here $u$ and $v$ are symbolized as velocity constituents $x$ and $y$ respectively. $U_{e}$ designated as free stream velocity, $v_{h n f}$ stands for kinematic viscosity of hybrid nanofluid, $\rho_{h n f}$ characterizes density of hybrid nanofluid, heat capacity of hybrid nanofluid is denoted by $\left(\rho C_{p}\right)_{h n f}, \alpha_{h n f}$ indicates thermal diffusivity of hybrid nanofluid. $T$ stands for fluid temperature.

Corresponding boundary conditions are

$u=U_{w}(x)=a x, v=0, T=T_{w}, \quad$ at $\quad y=0$,

$u=U_{e}(x)=b x, T \rightarrow T_{\infty}, \quad$ at $y \rightarrow \infty$.

Here $u$ and $v$ denotes velocity components, $U_{w}(x)(=a x)$ stands for stretching velocity , $T_{0}$ denotes refference temperature, $T_{w}$ is the temperature of plate, ambient temperature is represented by $T_{\infty}$.

Exploiting the transformations

$u=a x f^{\prime}(\eta), v=-\sqrt{a v} f(\eta), \eta=\sqrt{\frac{a}{v}} y, \theta(\eta)=\frac{T-T_{\infty}}{T_{w}-T_{0}}$,

Equation (1) is fulfilled consistently. Though Eqs. (2-5) appears as:

$$
\begin{aligned}
& f^{\prime \prime \prime}-B\left(1-\Phi_{1}\right)^{2.5}\left(1-\Phi_{2}\right)^{2.5}\left(\left(f^{\prime}\right)^{2}-f f^{\prime \prime}\right)-A^{2}=0 \\
& \left(\frac{B_{1}}{\operatorname{Pr}} \theta^{\prime \prime}+\lambda \theta\right) \frac{1}{D_{1}}+f \theta^{\prime}=0
\end{aligned}
$$


$f^{\prime}(0)=1, f(0)=0, f^{\prime}(\infty)=A$,

$\theta(0)=1, \theta(\infty)=0$,

In these equations $A$ denotes velocity ratio parameter, $\operatorname{Pr}$ symbolizes the Prandtl number, $\lambda$ is heat generation/absorption parameter. Algorithmic representation of these quantities are specified as :

$$
\begin{aligned}
A & =\frac{b}{a}, \operatorname{Pr}=\frac{v}{\alpha}, \lambda=\frac{Q}{\left(\rho C_{p}\right)_{f} a}, B_{1}=\frac{k_{h n f}}{k_{f}}, \\
B & =\left[\left(1-\Phi_{2}\right)\left\{\left(1-\Phi_{1}\right)+\Phi_{1} \frac{\rho_{s 1}}{\rho_{f}}\right\}+\Phi_{2} \frac{\rho_{s 2}}{\rho_{f}}\right], \\
D_{1} & =\left[\left(1-\Phi_{2}\right)\left\{\left(1-\Phi_{1}\right)+\Phi_{1} \frac{(\rho C)_{s 1}}{(\rho C)_{f}}\right\}+\Phi_{2} \frac{(\rho C)_{s 2}}{(\rho C)_{f}}\right],
\end{aligned}
$$

Mathematical form of drag force is prescribed as:

$$
C_{f}=\frac{\tau_{w}}{\rho U_{w}^{2}},
$$

Nondimensionalized configuiration is expressed as

$$
C_{f} \operatorname{Re}_{x}^{1 / 2}=-\frac{1}{\left(1-\Phi_{1}\right)^{2.5}\left(1-\Phi_{2}\right)^{2.5}} f^{\prime \prime}(0),
$$

Nusselt number is declared as

$$
N u=\frac{x q_{w}}{k_{f}\left(T_{w}-T_{\infty}\right)},
$$

Its undimensional form is as below

$$
N u \operatorname{Re}_{x}^{1 / 2}=-\frac{k_{h n f}}{k_{f}} \theta^{\prime}(0),
$$

here local Reynolds number is symbolized by $\operatorname{Re}_{x}=U_{w}(x) x / v$.

\section{Entropy generation}

Here our principal focus is to evaluate the irreversibilities of a system through entropy generation.Mathematically it is given as 


$$
E_{G}=\frac{k_{h n f}}{\left(T_{\infty}\right)^{2}}\left(\frac{\partial T}{\partial y}\right)^{2}+\frac{\mu_{h n f}}{T_{\infty}}\left(\frac{\partial u}{\partial y}\right)^{2},
$$

Dimensionless numerical formula for entropy generation is demonstrated as

$$
N_{s}=\frac{\left(T_{\infty}\right)^{2}\left(\frac{\eta}{y}\right)^{2}}{k_{h n f}\left(T_{w}-T_{\infty}\right)^{2}} E_{G},
$$

Achieved nondimensional form is

$$
N_{s}=\theta^{\prime 2}+\frac{E c \operatorname{Pr}}{\Omega\left(1-\Phi_{1}\right)^{2.5}\left(1-\Phi_{2}\right)^{2.5}} \frac{k_{f}}{k_{h n f}} f^{\prime \prime 2},
$$

Bejan number is expressed as

$$
\begin{aligned}
& B e=\frac{\text { Entropy generation due to thermal irreversibility }}{\text { Total entropy generation }}, \\
& B e=\frac{\theta^{\prime 2}}{\theta^{\prime 2}+\frac{E c \operatorname{Pr}}{\Omega\left(1-\Phi_{1}\right)^{2.5}\left(1-\Phi_{2}\right)^{2.5}} \frac{k_{f}}{k_{h n f}} f^{\prime 2}},
\end{aligned}
$$

\section{Homotopic solutions}

Homotopic method was exposed by Liao [33]. This method is utilized for finding the series solutions of highly non linear problems. Initial gusses and linear approximations are free to choose. They are intimated as follows:

$f_{0}(\eta)=A \eta-(A-1)(1-\exp (-\eta))$

$\theta_{0}(\eta)=\exp (-\eta)$

$\mathbf{L}_{f}(f)=\frac{d^{3} f}{d \eta^{3}}+\frac{d^{2} f}{d \eta^{2}}, \mathbf{L}_{\theta}(\theta)=\frac{d^{2} \theta}{d \eta^{2}}-\theta$,

With

$\mathbf{L}_{f}\left[\left(C_{1}+C_{2} \eta\right)+C_{3} \exp (-\eta)\right]=0$

$\mathrm{L}_{\theta}\left[C_{4} \exp (\eta)+C_{5} \exp (-\eta)\right]=0$,

here $C_{i}(i=1, \ldots, 5)$ characterizes the arbitrary constants.

The final solutions $\left(f_{m}, \theta_{m}\right)$ with the association of special solutions $\left(f_{m}^{*}(\eta), \theta_{m}^{*}(\eta)\right)$ are 
disclosed by

$f_{m}(\eta)=f_{m}^{?}(\eta)+C_{1}+C_{2} \eta+C_{3} e^{-\eta}$,

$\theta_{m}(\eta)=\theta_{m}^{?}(\eta)+C_{4} e^{\eta}+C_{5} e^{-\eta}$

\section{Convergence analysis}

Homotopy analysis method is that method which gave us freedom to choose and control the convergance region. Fig. 1. reflects the $\hbar$-curves for $f^{\prime}(\eta)$ and $\theta(\eta)$. The ranges for $\hbar_{f}$ and $\hbar_{\theta}$ are $-0.6 \leq \hbar_{f} \leq-0.1$ and $-.0 .8 \leq \hbar_{\theta} \leq-0.5$.

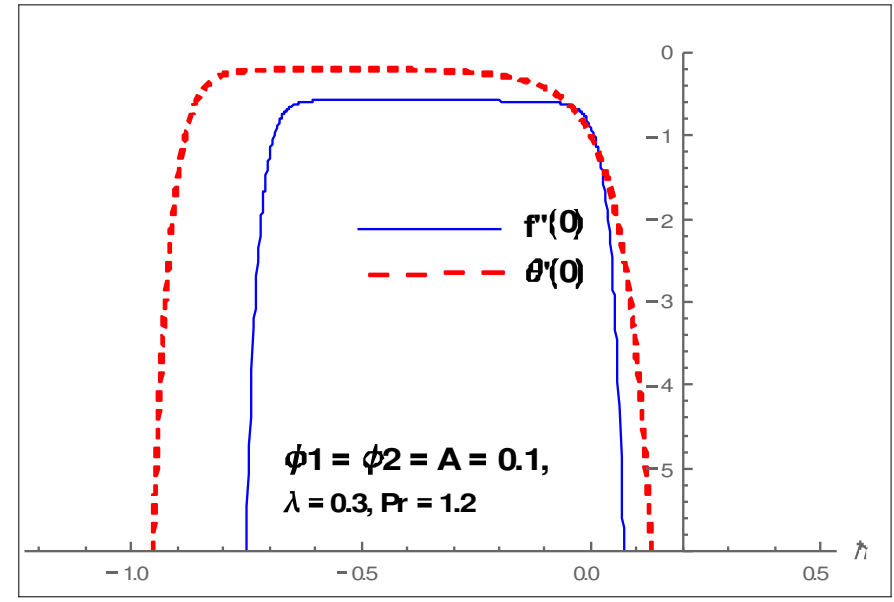

Fig. 1. h-Curve for $f(\eta)$ and $\theta(\eta)$.

\section{Discussion}

Graphical demonstration of influential parameters for flow and heat transmission are given in this portion.Thus figures are portrayed. Effect of velocity ratio parameter $A$ on velocity profile is illustrated in Fig.2. It is noticed that both velocity profile and momentum boundary layer thickness enhanced for increased velocity ratio parameter. Physically magnified ratio parameter prop up the free stream velocity which assists the velocity upgradation. Figs. (3-4). are plotted for polystyrene particles volume fraction $\Phi_{1}$ versus velocity and heat profiles. Opposite behavior is detected for velocity and temperature distribution. Velocity profile grows and temperature profile gets steeper. Fig. 5 depicts the behavior of titanium oxide $\left(\mathrm{TiO}_{2}\right)$ particles volume fraction $\Phi_{2}$ on velocity field. Titanium oxide nanoparticles decays the velocity field. Higher concentration of titanium oxide $\left(\mathrm{TiO}_{2}\right)$ particles provides significant resistance to the fluid consequently velocity deteriorate. Fig. 6. indicates the impact of titanium oxide $\left(\mathrm{TiO}_{2}\right)$ particles volume fraction $\Phi_{2}$ on temperature distribution. Alternate trend is obtained as for velocity field. Nano particles volume fraction strengthens the thermal conductivity of a base fluid. Therefore temperature distribution marked up. Fig. 7. indicates the influence of heat 
generation/absorption parameter $\lambda$ on temperature profile. Heat generation/ absorption parameter $\lambda$ strengths the thermal boundary layer and temperature profile. In the course of heat generation activity extra heat will be generated and eventually temperature distribution boost. Out turn of Prandtl number on temperature field is displayed in fig. 8. Temperature profile diminishes for intensified Prandtl number Pr. Physically thermal diffusivity fall down for upgraded Prandtl number Pr. So we obtain narrow temperature profile. Figs. (9-10). displays the heat transfer rate versus Prandtl number $\operatorname{Pr}$ and heat generation parameter $\lambda$. It is observed that heat transfer rate enhances for Prandtl number $\operatorname{Pr}$ as well as heat generation/absorption parameter $\lambda$. Physically higher Prandtl number reduces the temperature field. Therefore maximum amount of heat will be transferred to the environment. As a result rate of heat transfer increases. Fig. 10. expresses the 3D plot of nusselt number for pertinent parameter. Increment in heat transfer rate has been detected. The reason for this is that enlarged Prandtl number Pr declines the thermal boundary layer which inturns enhances heat transfer rate.

\section{Entropy production}

Figs. (11-15) . reflects the influences of various parameters on entropy generation. Fig. (11-12). represents the effect of polstyrene particles volume fraction $\Phi_{1}$ and titanium oxide particles volume fraction $\Phi_{2}$ on entropy generation. Results shows that both polystrene particles volume fraction and titanium oxide particles volume fraction enhances the entropy generation rate. To determine the impact of Prandtl number $\operatorname{Pr}$ and Eckert number $E c$ on entropy generation, figs. (13-14). are sketched. It is noticed that entropy generation rate is a increasing function of Prandtl as well as Eckert number. Actually due to enlarged Eckert number $E c$ supplementary heat will be induced and therefore entropy generation dominates. Fig. 15. portrayed the footprints of temperature difference parameter $\Omega$ on entropy production rate. Opposite behavior has been perceived as compared to Eckert number. Entropy generation rate diminishes when temperature difference parameter grows.

Table. 1 represents numerical values of thermophysical properties of Polystyrene and Titanium Oxide particles (nano particles) and table. 2 express the mathematical formulation of hybrid nanofluid and base fluid.

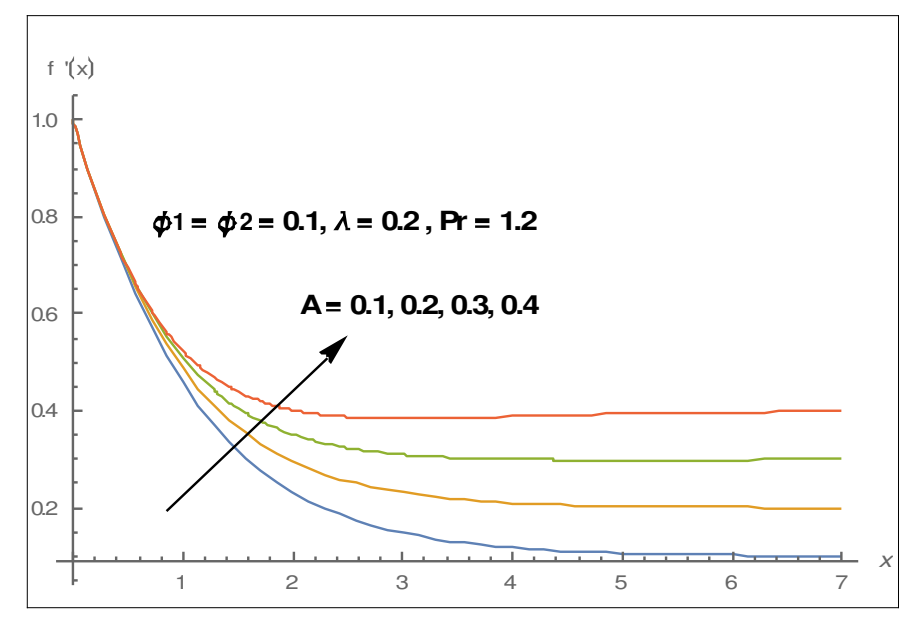

Fig. 2. Illustration of $A$ on $f^{\prime}(\eta)$.

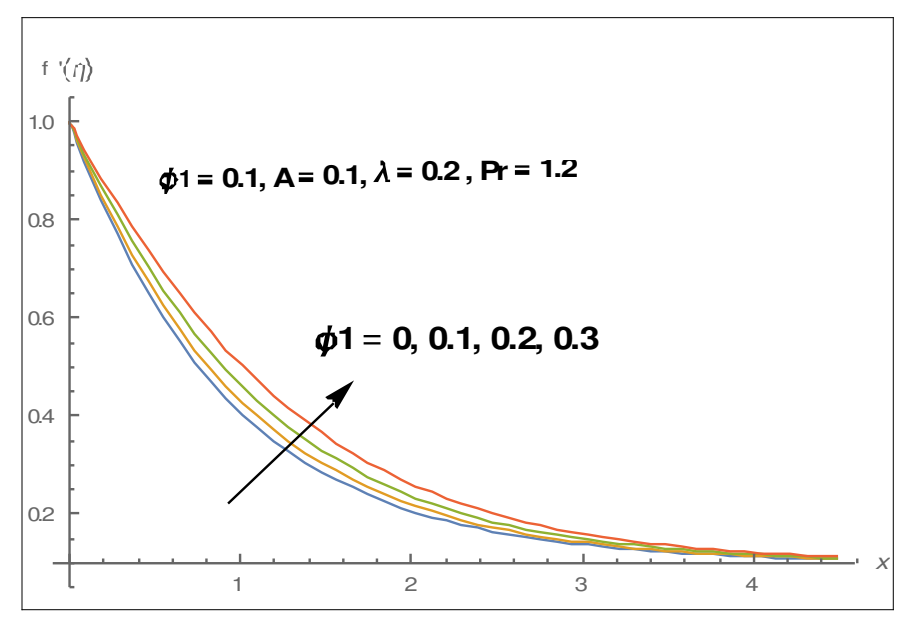

Fig. 3. Illustration of $\Phi_{1}$ on $f^{\prime}(\eta)$. 


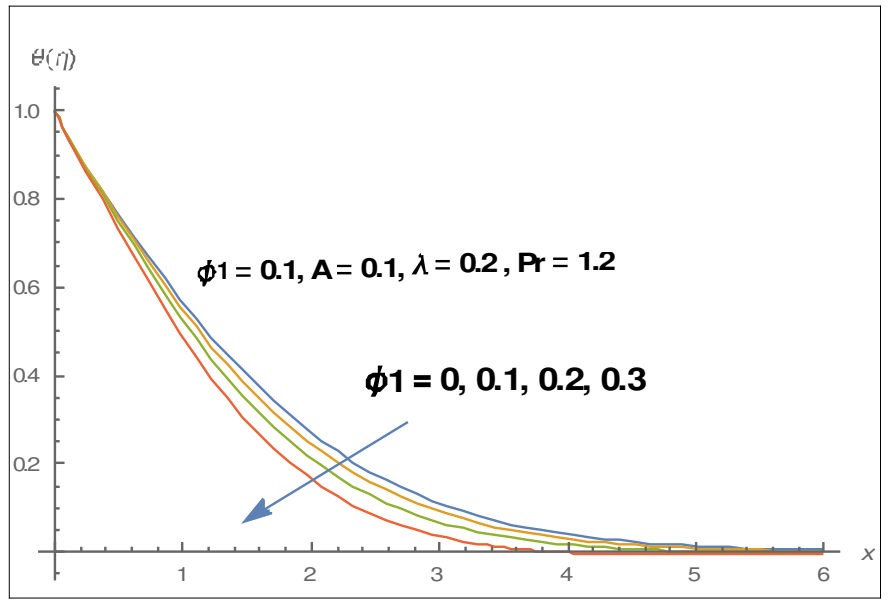

Fig. 4. Illustration of $\Phi_{1}$ on $\theta(\eta)$.

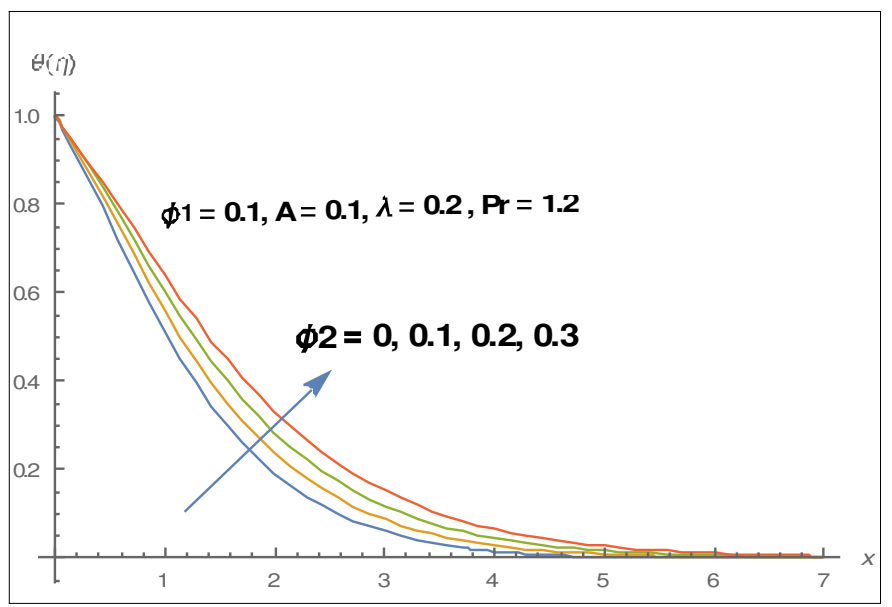

Fig. 6. Illustration of $\Phi_{2}$ on $\theta(\eta)$.

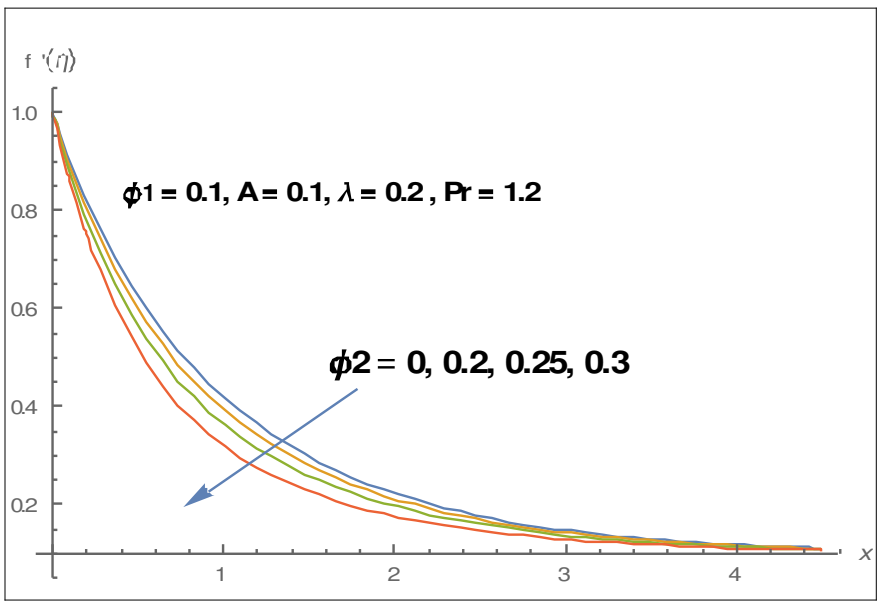

Fig. 5. Illustration of $\Phi_{2}$ on $f^{\prime}(\eta)$.

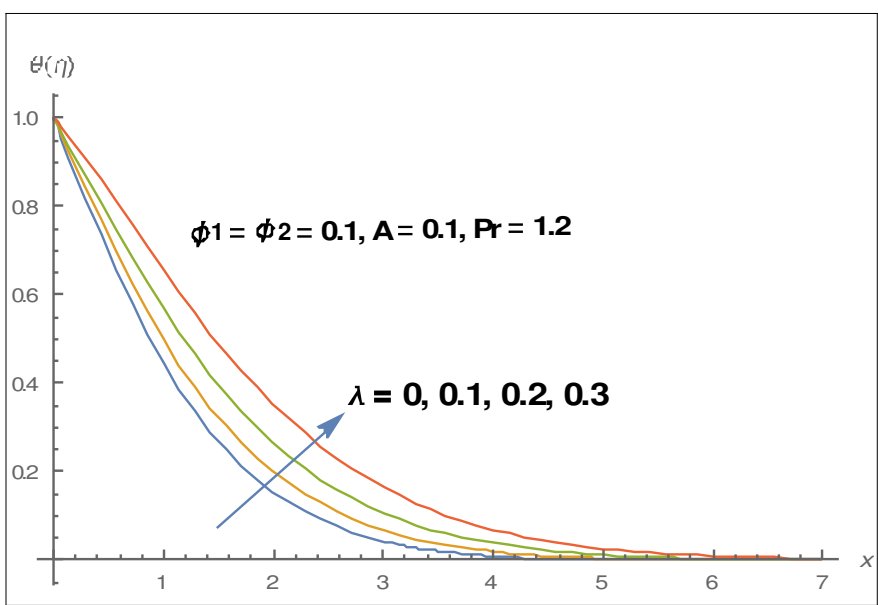

Fig. 7. Illustration of $\lambda$ on $\theta(\eta)$. 


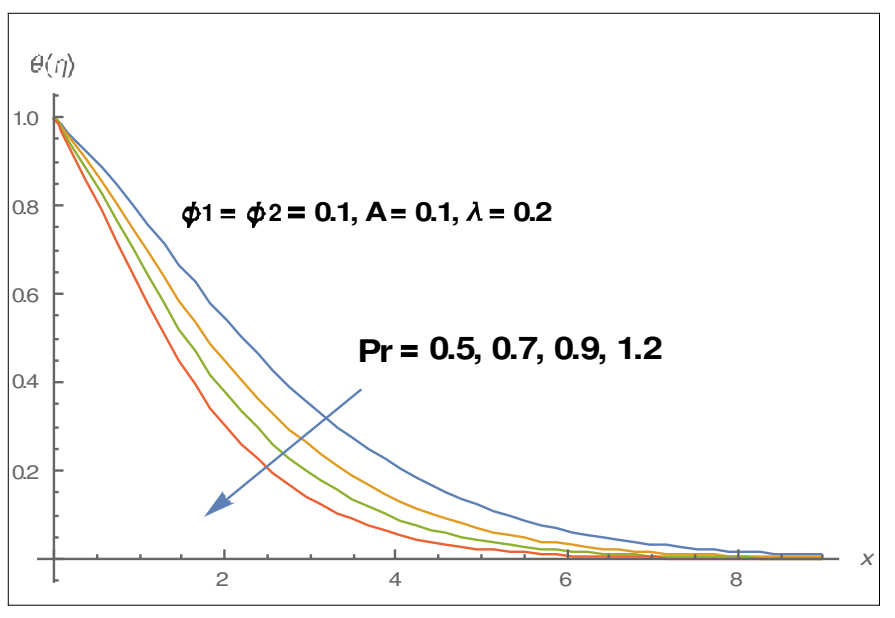

Fig. 8. Illustration of $\operatorname{Pr}$ on $\theta(\eta)$.

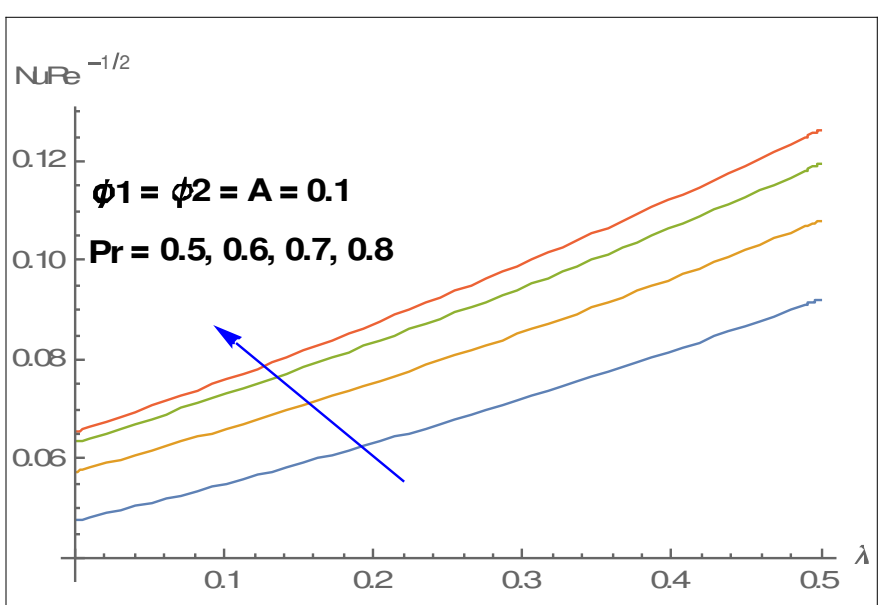

Fig. 9. Illustration of $\operatorname{Pr}$ and $\lambda$ for Heat transfer.

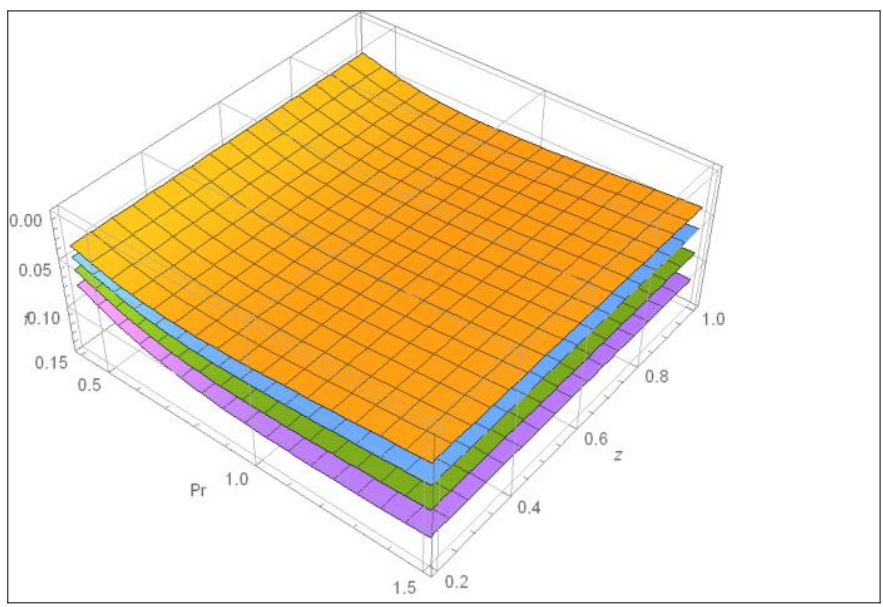

Fig. 10. Illustration of $\operatorname{Pr}$ and $\lambda$ for Nusselt number. 


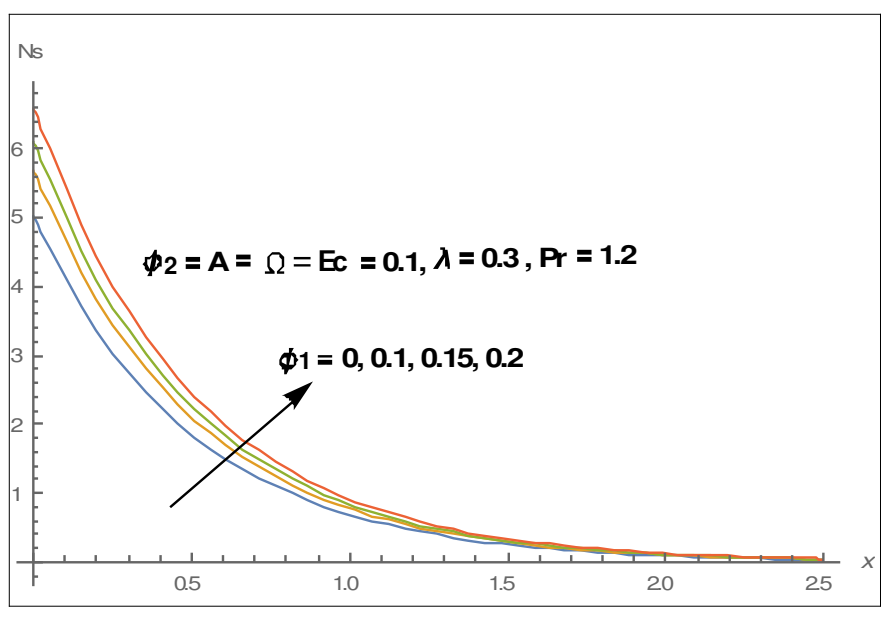

Fig. 11. Illustration of $\Phi_{1}$ on $N_{s}$.

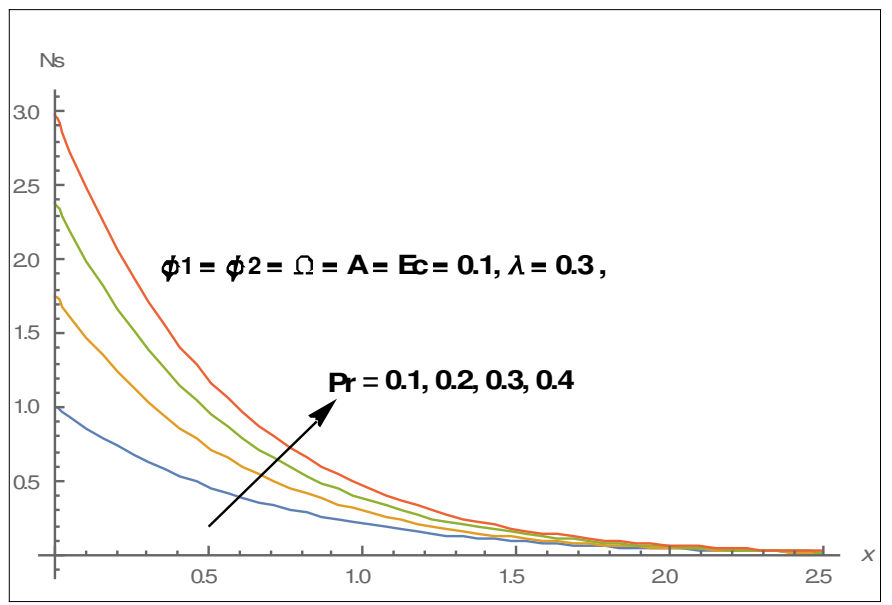

Fig. 13. Illustration of $\operatorname{Pr}$ on $N_{s}$.

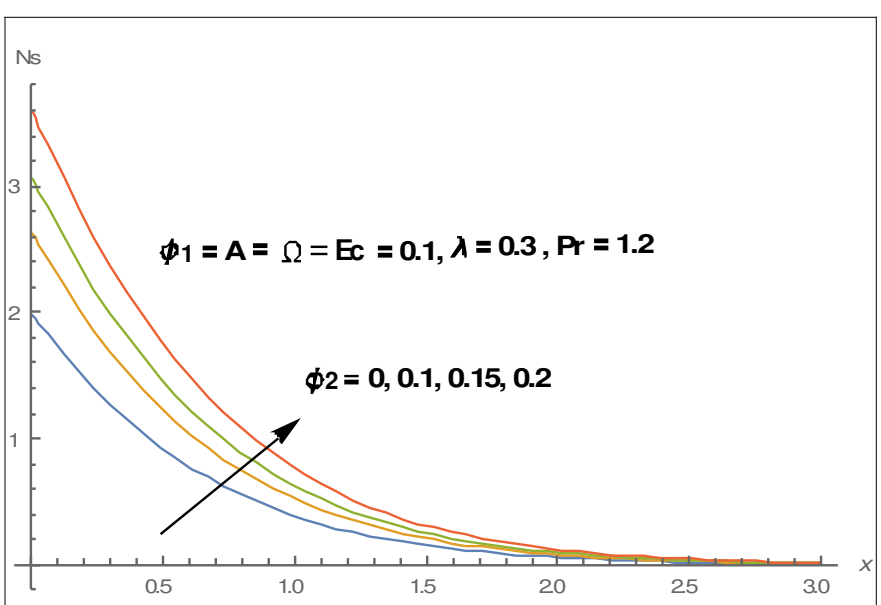

Fig. 12. Illustration of $\Phi_{2}$ on $N_{s}$.

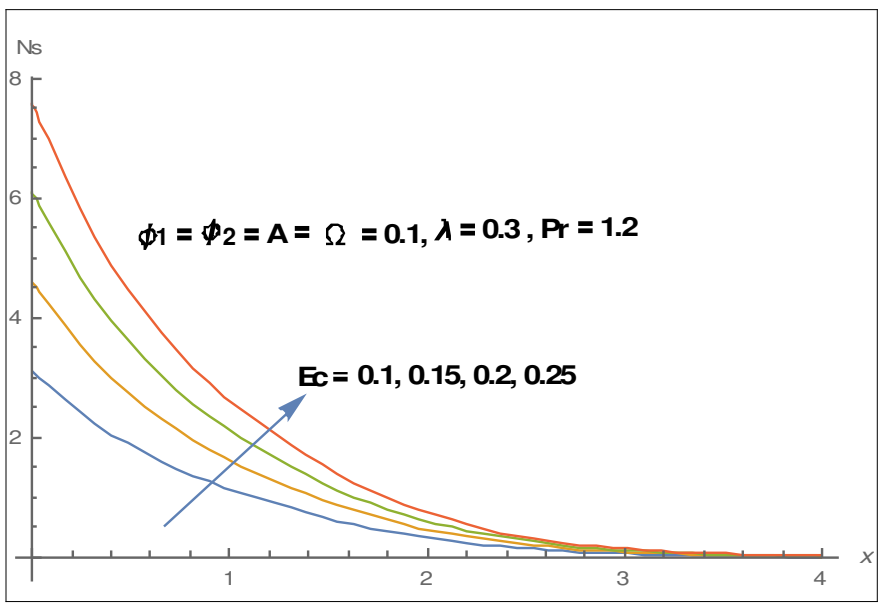

Fig. 14. Illustration of $E c$ on $N_{s}$. 


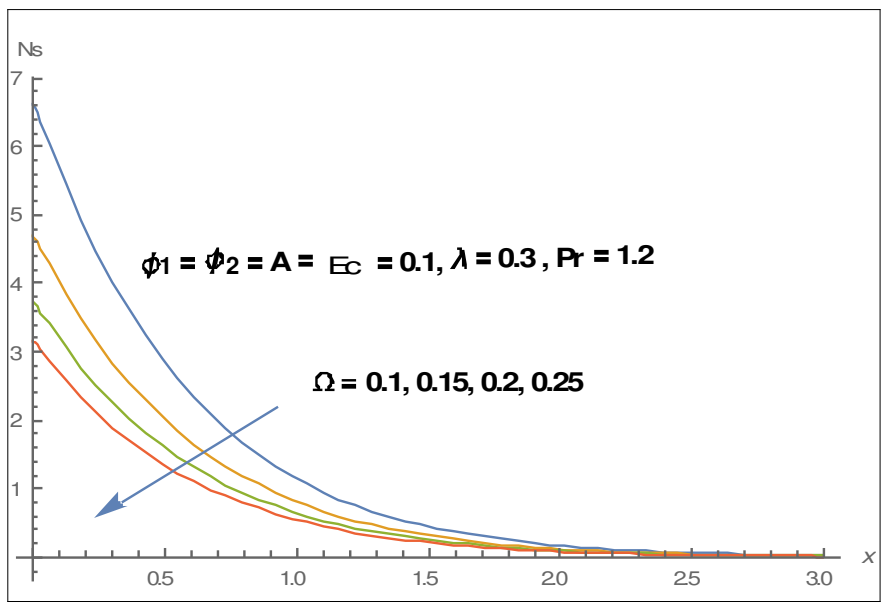

Fig. 15. Illustration of $\Omega$ on $N_{s}$.

Table 1: Thermophysical properties of base fluid and nano particles [40-41]:

\begin{tabular}{|l|l|l|l|}
\hline $\begin{array}{l}\text { Base fluid/Nano } \\
\text { particles }\end{array}$ & $\rho\left(\mathrm{kgm}^{-3}\right)$ & $C_{p}\left(\mathrm{JK}^{-1}\right)$ & $k\left(\mathrm{WK}^{-1} \mathrm{~m}^{-1}\right)$ \\
\hline Water $\left(\mathrm{H}_{2} \mathrm{O}\right)$ & 997.1 & 4179 & 0.613 \\
\hline Polystyrene & 1053 & 1210 & 0.16 \\
\hline $\begin{array}{l}\text { Titanium } \\
\text { Oxide }\left(\mathrm{TiO}_{2}\right)\end{array}$ & 4250 & 686.2 & 8.9538 \\
\hline
\end{tabular}

Table 2: Mathematical formulation of thermophysical properties of hybrid nanofluid $[42-45]$ :

\begin{tabular}{|c|c|}
\hline Properties & Hybrid Nanofluid \\
\hline Dynamic Viscocity $\left(\mathrm{Nsm}^{-2}\right)$ & $\mu_{h n f}=\frac{\mu_{f}}{\left(1-\Phi_{1}\right)^{2.5}\left(1-\Phi_{2}\right)^{2.5}}$ \\
\hline Density $\left(\mathrm{kgm}^{-3}\right)$ & $\rho_{h n f}=\left[\left(1-\Phi_{2}\right)\left\{\left(1-\Phi_{1}\right) \rho_{f}+\Phi_{1} \rho_{s 1}\right\}+\Phi_{2} \rho_{s 2}\right]$ \\
\hline \multirow[t]{3}{*}{ Thermal Conductivity $\left(\mathrm{WK}^{-1} m^{-1}\right)$} & $\frac{k_{h n f}}{k_{n f}}=\frac{k_{s 2}+(n-1) k_{n f}-(n-1) \Phi_{2}\left(k_{n f}-k_{s 2}\right)}{k_{s 2}+(n-1) k_{n f}+\Phi_{2}\left(k_{n f}-k_{s 2}\right)}$ \\
\hline & where \\
\hline & $\frac{k_{n f}}{k_{f}}=\frac{k_{s 1}+(n-1) k_{f}-(n-1) \Phi_{1}\left(k_{f}-k_{s 1}\right)}{k_{s 1}+(n-1) k_{f}+\Phi_{1}\left(k_{f}-k_{s 1}\right)}$ \\
\hline Heat Capacity $\left(J K^{-1}\right)$ & {$\left[\left(1-\Phi_{2}\right)\left\{\left(1-\Phi_{1}\right)\left(\rho C_{p}\right)_{f}+\Phi_{1}\left(\rho C_{p}\right)_{s 1}\right\}+\Phi_{2}\left(\rho C_{p}\right)_{s 2}\right]$} \\
\hline
\end{tabular}


Properties

Dynamic viscosity $\left(\mathrm{Nsm}^{-2}\right)$

Density $\left(\mathrm{kgm}^{-3}\right)$
Hybrid Nanofluid

$$
\begin{gathered}
\mu_{h n f}=\frac{\mu_{f}}{\left(1-\Phi_{1}\right)^{2.5}\left(1-\Phi_{2}\right)^{2.5}} \\
\rho_{h n f}=\left[\left(1-\Phi_{2}\right)\left\{\left(1-\Phi_{1}\right) \rho_{f}+\Phi_{1} \rho_{s 1}\right\}+\Phi_{2} \rho_{s 2}\right] \\
\frac{k_{h n f}}{k_{n f}}=\frac{k_{s_{2}}+(n-1) k_{n f}-(n-1) \Phi_{2}\left(k_{n f}-k_{s_{2}}\right)}{k_{s_{2}}+(n-1) k_{n f}+\Phi_{2}\left(k_{n f}-k_{s_{2}}\right)}
\end{gathered}
$$

Thermal Conductivity $\left(W K^{-1} m^{-1}\right)$

where

$$
\frac{k_{n f}}{k_{f}}=\frac{k_{s_{1}}+(n-1) k_{f}-(n-1) \Phi_{1}\left(k_{f}-k_{s_{1}}\right)}{k_{s 1}+(n-1) k_{f}+\Phi_{1}\left(k_{f}-k_{s_{1}}\right)}
$$

Heat Capacity $\left(J K^{-1}\right)$

\section{Conclusions}

Influence of heat generation/absorption and stagnation point on hybrid nanofluid are taken into account. Hybrid nanofluid contains Polystyrene and $\mathrm{TiO}_{2}$ nanoparticles with water as a transiet fluid. The disruptive results for the sophisticated parameters are displayed graphically. The majour outcomes are as persued:

- Volume fraction of polystyrene particles $\Phi_{1}$ enlarged the velocity profile and degrade the temperature profile.

- Volume fraction of titanium oxide $\Phi_{2}$ particles decline the velocity field whereas adverse behavior is detected for temperature field.

- Velocity ratio parameter enhanced the velocity field.

- Heat generation/absosption parameter emlarged the temperature profile.

- Entropy generation intensifies for both polystyrene and titanium oxide parcticles.

- Prandtl number Pr and Eckert number Ec also amplifies the entropy generation strength.

- Temperature difference parameter decays the entropy generation.

The preference is that the contemporaneous analysis will be extremely beneficial for modeling better flow obstacles specifically in biomedical industary, for cancer treatment, aerodynamic industary, power generation, nuclear reactors and solar thermal absorbers.

\section{References}

(1). Choi, SUS: Enhancing thermal conductivity of fluids with nanoparticles. In: The Proceedings of the ASME International Mechanical Engineering Congress and Exposition (San Francisco, USA, ASME, FED, 231/MD), 66 (1995): 99-105.

(2). I. Waini, A. Ishak \& I. Pop. (2020). Transpiration effects on hybrid nanofluid flow and heat transfer over a stretching/shrinking sheet with uniform shear flow. Alexandria Engineering Journal. 59, 91--99.

(3). A. Tassaddiq, S. Khan, M. Bilal, T. Gul, S. Mukhtar, Z. Shah \& E. Bonyah. (2020). Heat and mass transfer together with hybrid nanofluid flow over a rotating disk. AIP Advances. 10, doi.org/10.1063/5.0010181.

(4). T. Tayebi, H. F. Öztop \& A. J. Chamkha. (2020). Natural convection and entropy production 
in hybrid nanofluid filled-annular elliptical cavity with internal heat generation or absorption. Thermal Science and Engineering Progress, doi.org/10.1016/j.tsep.2020.100605.

(5). U. M. Zahid, Y. Akbar \& F.M. Abbasi. (2020). Entropy generation analysis for peristaltically driven flow of hybrid nanofluid. Chinese Journal of Physics. 67, 330--348.

(6). T. A. Yusuf, F. Mabood, W.A. Khan \& J.A. Gbadeyan. (2020). Irreversibility analysis of $\mathrm{Cu}-\mathrm{TiO} 2-\mathrm{H} 2 \mathrm{O}$ hybrid-nanofluid impinging on a 3-D stretching sheet in a porous medium with nonlinear radiation: Darcy-Forchhiemer's model. Alexandria Engineering Journal, 59, 5247-5261.

(7). V.V. Wanatasanapan, M.Z. Abdullah \& P. Gunnasegaran. (2020). Effect of TiO2-A12O3 nanoparticle mixing ratio on the thermal conductivity, rheological properties, and dynamic viscosity of water-based hybrid nanofluid. Journal od Material Research and Technology, 9, 13781-13792.

(8). Z. Said, M. Ghodbane, L. S. Sundar, A. K. Tiwari, M. Sheikholeslami \& B. Boumeddane. (2020). Heat transfer, entropy generation, economic and environmental analyses of linear Fresnel reflector using novel rGO-Co3O4 hybrid nanofluids. Renewable Energy, doi.org/10.1016/j.renene.2020.11.054 .

(9). N. Abbas , S. Nadeem , A. Saleem , M.Y. Malik ,A. Issakhov \& F. M. Alharabi. (2020).Models base study of inclined MHD of hybrid nanofluid flow over nonlinear stretching cylinder. Chinese Journal of Physics, doi.org/10.1016/j.cjph.2020.11.019.

(10). A. A. A. Arani \& H. Aberoumand. (2020). Stagnation-point flow of Ag-CuO/water hybrid nanofluids over a permeable stretching/shrinking sheet with temporal stability analysis. Powder Technology, doi.org/10.1016/j.powtec.2020.11.043.

(11). X. Li , N. Indumathi, H. Wang \& B. Luo. (2020). The thermophysical properties and enhanced heat transfer performance of SiC-MWCNTs hybrid nanofluids for car radiator system. Colloids and Surfaces A: Physicochemical and Engineering Aspects, doi.org/10.1016/j.colsurfa.2020.125968.

(12). N. A. Zainal , R. Nazar , K. Naganthran \& I. Pop. (2020) . Heat generation/absorption effect on MHD flow of hybrid nanofluid over bidirectional exponential stretching/shrinking sheet. Chinese Journal of Physics, doi.org/10.1016/j.cjph.2020.12.002.

(13). K. Naganthran, R. Nazara \& I. Pop.(2017). Stability analysis of impinging oblique stagnation-point flow over a permeable shrinking surface in a viscoelastic fluid. International Journal of Mechanical Sciences, 131, 663-671.

(14). M. I. Khan, T. Hayat, F. Shah, M. Rahman \& F. Haq. (2019). Physical aspects of CNTs and induced magnetic flux in stagnation point flow with quartic chemical reaction. International Journal of Heat and Mass Transfer. 135, 561-568.

(15). J. Ahmed, M. Khan \& L. Ahmad. (2019). Stagnation point flow of Maxwell nanofluid over a permeable rotating disk with heat source/sink. Journal of Molecular Liquids. 287, 110853.

(16). N. P. Moshkin, V.V. Pukhnachev \& Yu. D. Bozhkov. (2019). On the unsteady, stagnation point flow of a Maxwell fluid in 2D. International Journal of Non-Linear Mechanics, 116, 32-38. (17). P. Weidman. (2019). Hiemenz stagnation-point flow impinging on a uniformly rotating plate. European Journal of Mechanics / B Fluids, 78, 169-173.

(18). T.R. Mahapatra \& S. Sidui. (2019). Non-axisymmetric Homann stagnation-point flow of a viscoelastic fluid towards a fixed plate. European Journal of Mechanics / B Fluids, 79, 38-43.

(19). E. Azhar, Z. Iqbal \& E. N. Maraj.(2019). Viscous dissipation performance on stagnation point flow of Jeffrey fluid inspired by internal heat generation and chemical reaction. Thermal Science and Engineering Progress. 13, 100377. 
(20). Y. M. Chu, M. I. Rehman, M. I. Khan, S. Nadeem, S. Kadry, Z. Abdelmalekh \& N. Abbas. (2020). Transportation of heat and mass transport in hydromagnetic stagnation point flow of Carreau nanomaterial: Dual simulations through Runge-Kutta Fehlberg technique. International Communications in Heat and Mass Transfer, 118, 104858.

(21). F. Shah, M. I. Khan, T. Hayat, S. Momani \& M. I. Khan. (2020). Cattaneo-Christov heat flux (CC model) in mixed convective stagnation point flow towards a Riga plate. Computer Methods and Programs in Biomedicine. doi.org/10.1016/j.cmpb.2020.105564.

(22). A. U. Awan, S. Abid, N. Ullah \& S. Nadeem. (2020). Magnetohydrodynamic oblique stagnation point flow of second grade fluid over an oscillatory stretching surface. Results in Physics, 18, 103233.

(23). A. Bejan. (1979). A study of entropy generation in fundamental convective heat transfer. Journal of Heat Transfer, 101, 718--725.

(24). P. Gholamalipour, M. Siavashi \& M. H. Doranehgard. (2019). Eccentricity effects of heat source inside a porous annulus on the natural convection heat transfer and entropy generation of $\mathrm{Cu}$-water nanofluid. International Communications in Heat and Mass Transfer. 109, 104367.

(25). S. Dutta, N. Goswami, A. K. Biswas \& S. Pati. (2019). Numerical investigation of magnetohydrodynamic natural convection heat transfer and entropy generation in a rhombic enclosure filled with $\mathrm{Cu}$-water nanofluid. International Journal of Heat and Mass Transfer. 136, 777- 798.

(26). A. Khan, Z. Shah, E. Alzahrani \& S. Islam. (2020). Entropy generation and thermal analysis for rotary motion of hydromagnetic Casson nanofluid past a rotating cylinder with Joule heating effect. International Communications in Heat and Mass Transfer. 119, 104979.

(27). A. Ambreen, A. Saleem \& C. W. Park. (2020). Analysis of hydro-thermal and entropy generation characteristics of nanofluid in an aluminium foam heat sink by employing Darcy-Forchheimer-Brinkman model coupled with multiphase Eulerian model. Applied Thermal Engineering. 173, 115231.

(28). C. C. Cho. Effects of porous medium and wavy surface on heat transfer and entropy generation of $\mathrm{Cu}$-water nanofluid natural convection in square cavity containing partially-heated surface. International Communications in Heat and Mass Transfer. 119, 104925.

(29). T. Tayebi, H. F. Öztop \& A. J. Chamkha. (2020). Natural convection and entropy production in hybrid nanofluid filled-annular elliptical cavity with internal heat generation or absorption. Thermal Science and Engineering Progress. doi.org/10.1016/j.tsep.2020.100605.

(30). U.M. Zahid, Y. Akbar \& F.M. Abbasi. (2020). Entropy generation analysis for peristaltically driven flow of hybrid nanofluid. Chinese Journal of Physics. 67, 330-348.

(31). Z. Li, A. K. Hussein, O. Younis, M. Afrand \& S. Feng. (2020). Natural convection and entropy generation of a nanofluid around a circular baffle inside an inclined square cavity under thermal radiation and magnetic field effects . International Communications in Heat and Mass Transfer. 116, 104650.

(32). D. Sáchica, C. Treviño \& L. M. Suástegui. (2020). Numerical study of magnetohydrodynamic mixed convection and entropy generation of $\mathrm{Al} 2 \mathrm{O} 3$-water nanofluid in a channel with two facing cavities with discrete heating. International Journal of Heat and Fluid Flow. 86, 108713.

(33). S. J. Liao. (2009). Notes on the Homotopy Analysis Method: Some definitions and theorem. Communication in Nonlinear Science and Numerical Simulation, 14, 983-997.

(34). T. Hayat, S. Qayyum, M. Imtiaz \& A. Alsaedi. (2017). Flow between two stretchable rotating disks with Cattaneo-Christov heat flux model. Results in physics, 7, 126-133. 
(35). M. R. Shirkhani, H. A. Hoshyara, I. Rahimipetroudi, H. Akhavan \& D. D. Ganji. (2018). Unsteady time-dependent incompressible Newtonian fluid flow between two parallel plates by homotopy analysis method (HAM), homotopy perturbation method (HPM) and collocation method (CM). Propulsion and Power Research, 7, 247-256.

(36). A. Jafarimoghaddam. (2018). On the Homotopy Analysis Method (HAM) and Homotopy Perturbation Method (HPM) for a nonlinearly stretching sheet flow of Eyring-Powell fluids. Engineering Science and Technology, an International Journal, 22, 439-451.

(37). S. Masood, M. Farooq, S. Ahmad, A. Anjum \& N.A. Mir. (2019). Investigation of viscous dissipation in the nanofluid flow with aForchheimer porous medium: Modern transportation of heat and mass. European Physical Journal Plus. 134:178.

(38). S. Ahmad, M. Farooq, M. Rizwan, B. Ahmad \& S. Rehman. (2020). Melting Phenomenon in a Squeezed Rheology of Reactive Rate Type Fluid. Frontiers in Physics. doi.org/10.3389/fphy.2020.00108.

(39). S. Masood \& M. Farooq. (2021). Influence of thermal stratification and thermal radiation on graphene oxide- $\mathrm{Ag} / \mathrm{H} 2 \mathrm{O}$ hybrid nanofluid. Journal of Thermal Analysis and Calorimetry. 143, 1361-1370..

(40). M. Sheikholeslami, M. Hatami \& D.D. Ganji. (2014). Nanofluid flow and heat transfer in a rotating system in the presence of a magnetic field. Journal of Molecular Liquids, 190, 112-120.

(41). M. I. Khan. M. U. Hafeez, T. Hayat, M. I. Khan \& A. Alsaedi. (2020). Magneto rotating flow of hybrid nanofluid with entropy generation. Computers Medicines and Programs in Biomedicine, 183, 105093.

(42). J. Sarkar , P. Ghosh , A. Adil. (2015). A review on hybrid nanofluids: recent research, development and applications, Renew and Sustainable Energy Reviews, 43 (2015) 164--177 . (43). L. S. Sundar , K. V. Sharma , M. K. Singh \& A. C. M. Sousa. (2017). Hybrid nanofluids preparation, thermal properties, heat transfer and friction factor--a review.Renew and Sustainable Energy Reviews, 68, 185--198 .

(44). N. A. C. Sidik , M. M. Jamil , W. M. A . A . Japar \& I. M. Adamu. (2017). A review on preparation methods, stability and applications of hybrid nanofluids. Renew and Sustainable Energy Reviews, 80, 1112--1122 .

(45). M. Afrand , K. N. Najafabadi \& M. Akbari. (2016). Effects of temperature and solid volume fraction on viscosity of SiO 2 -MWCNTs / SAE 40 hybrid nanofluid as a coolant and lubricant in heat engines. Applied Thermal Engineering. 102 (2016) 45--54 . 
Figures

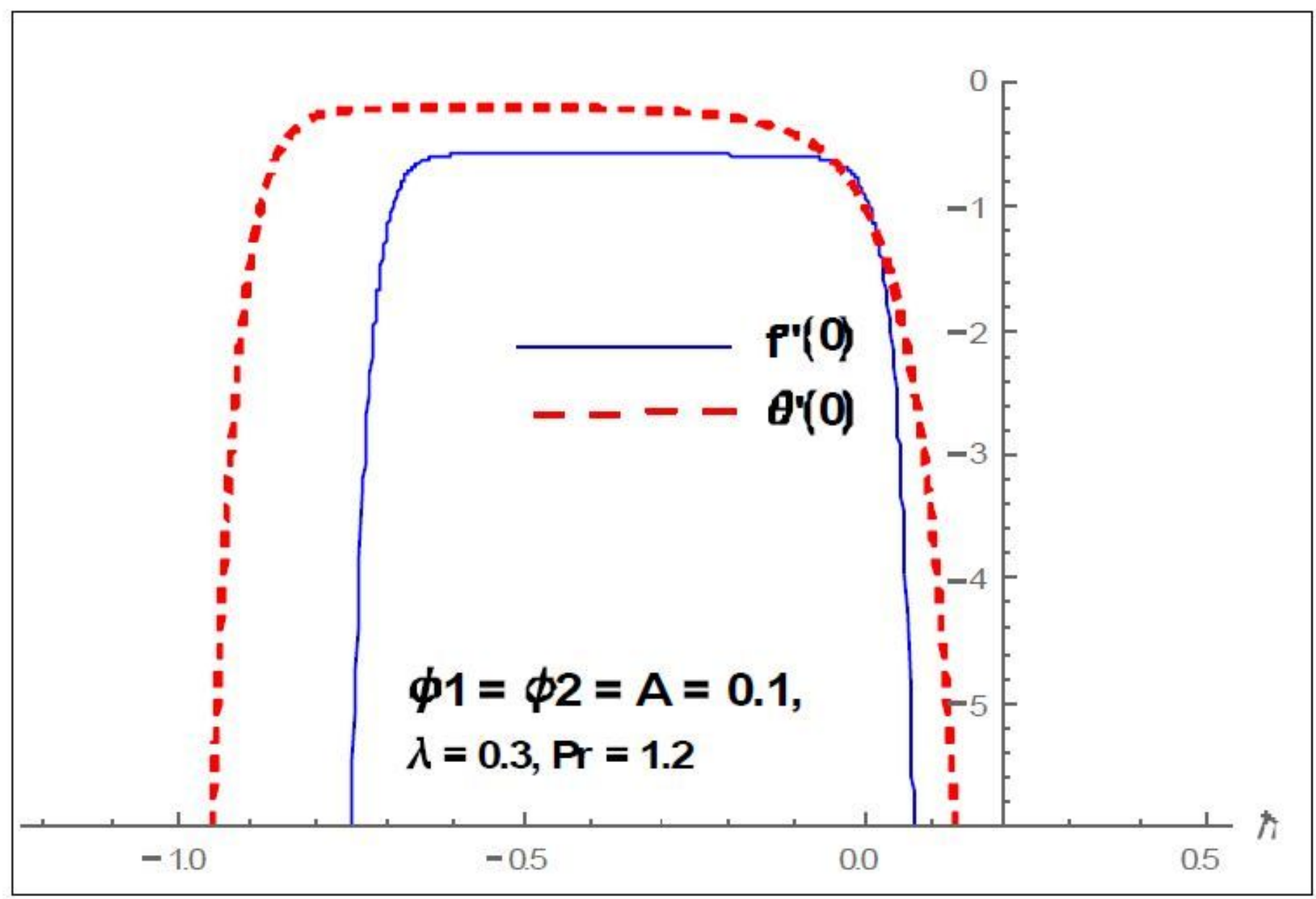

Figure 1

Due to technical limitations, figure captions can be found in the manuscript file. 


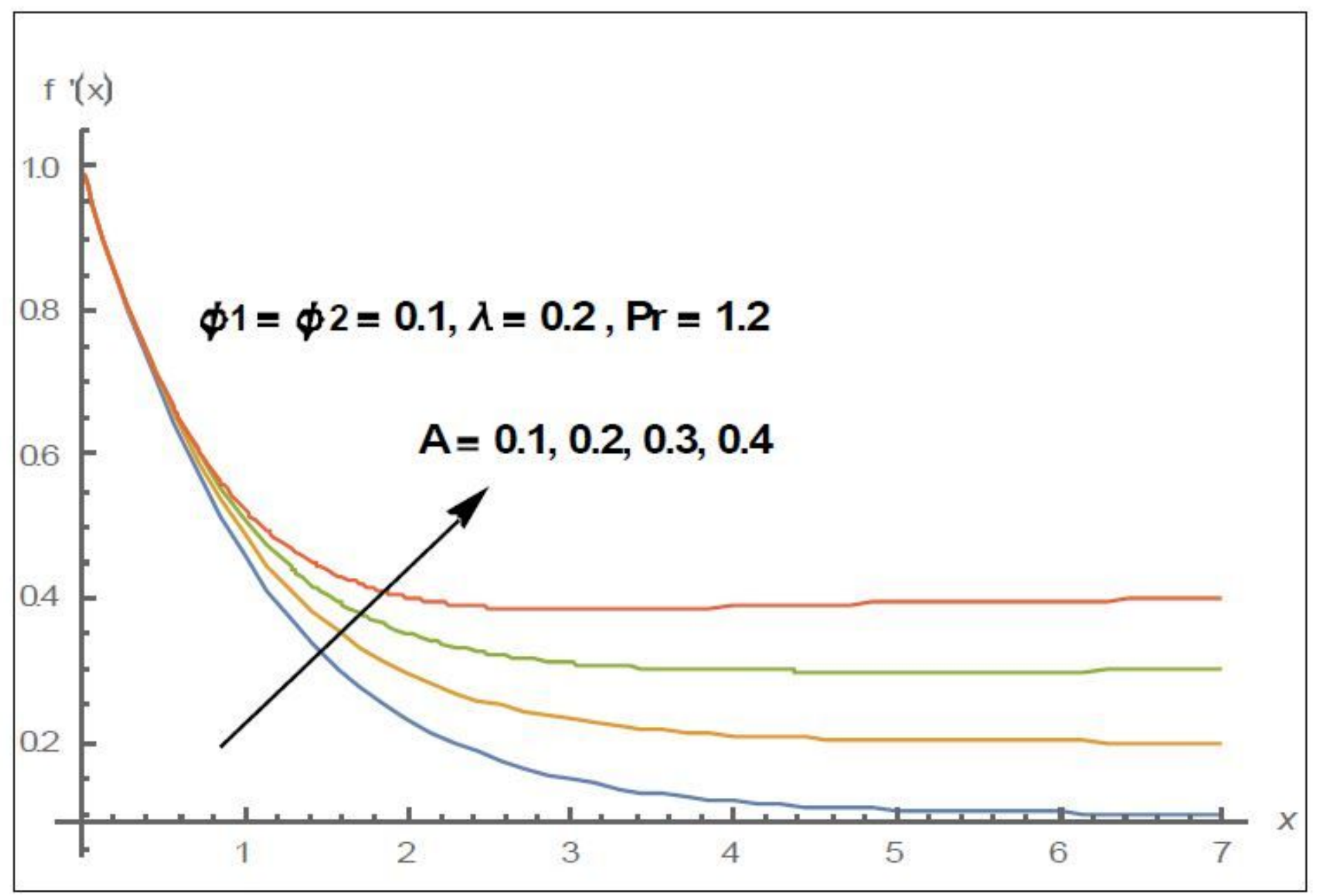

Figure 2

Due to technical limitations, figure captions can be found in the manuscript file. 


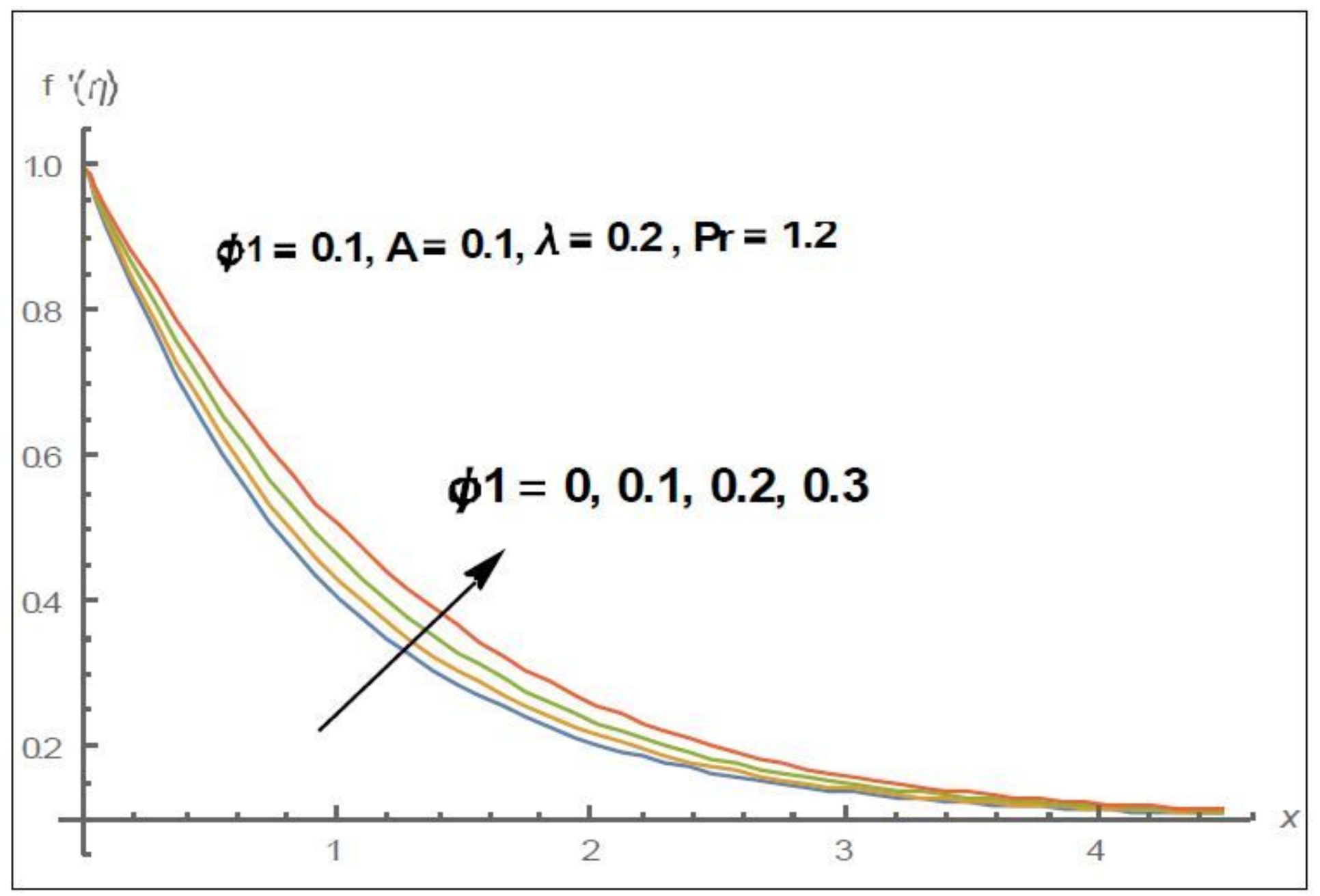

Figure 3

Due to technical limitations, figure captions can be found in the manuscript file. 


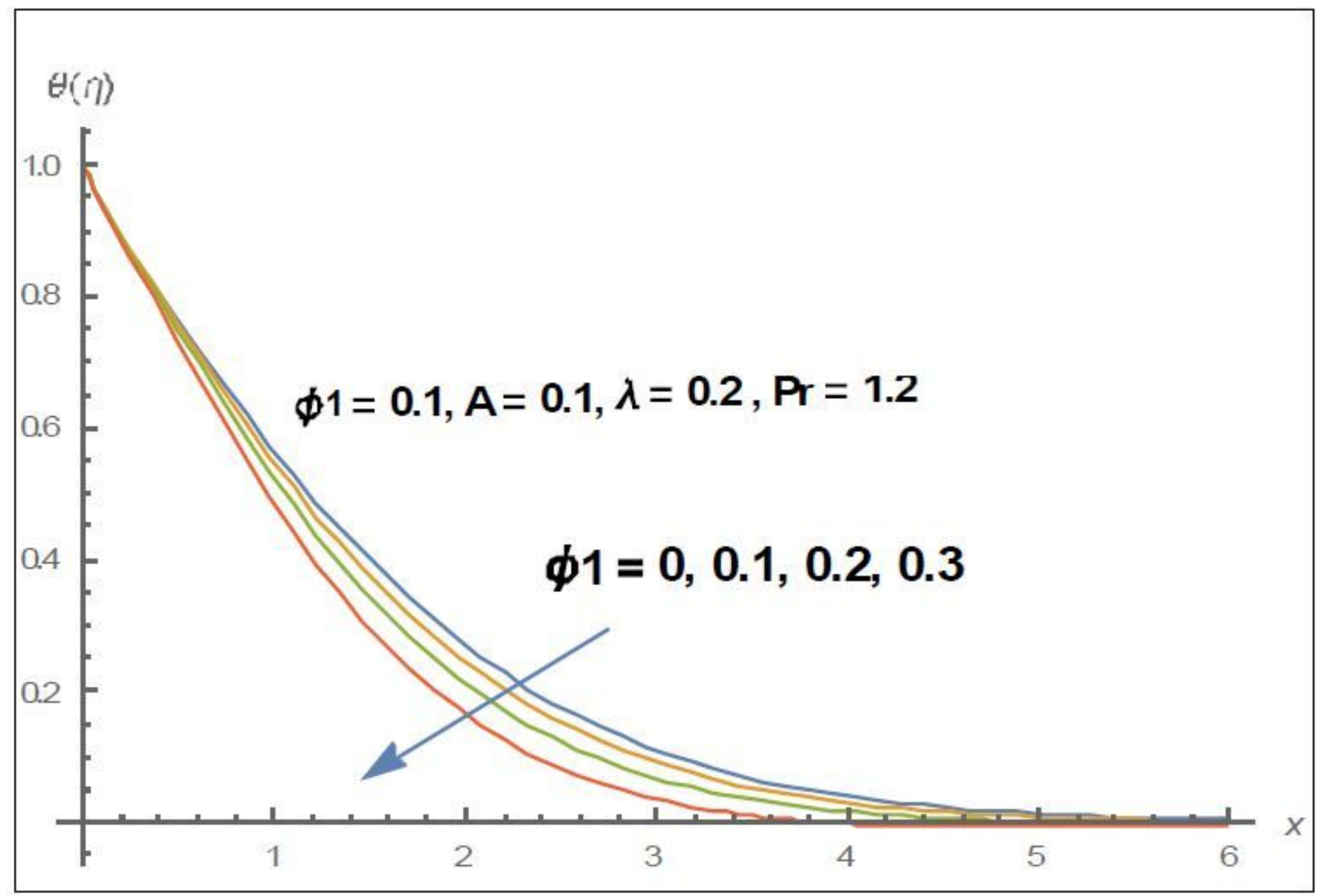

Figure 4

Due to technical limitations, figure captions can be found in the manuscript file. 


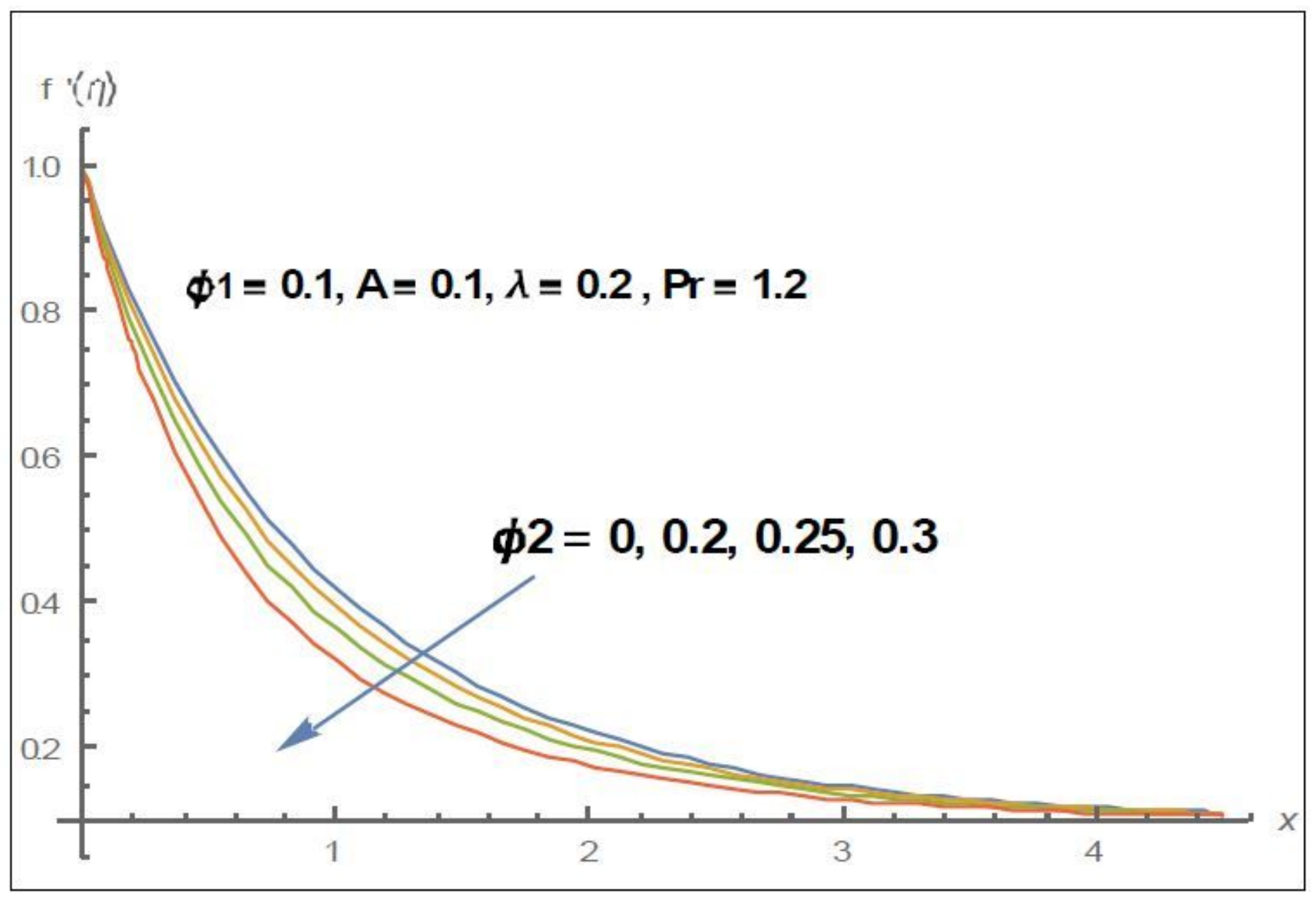

Figure 5

Due to technical limitations, figure captions can be found in the manuscript file. 


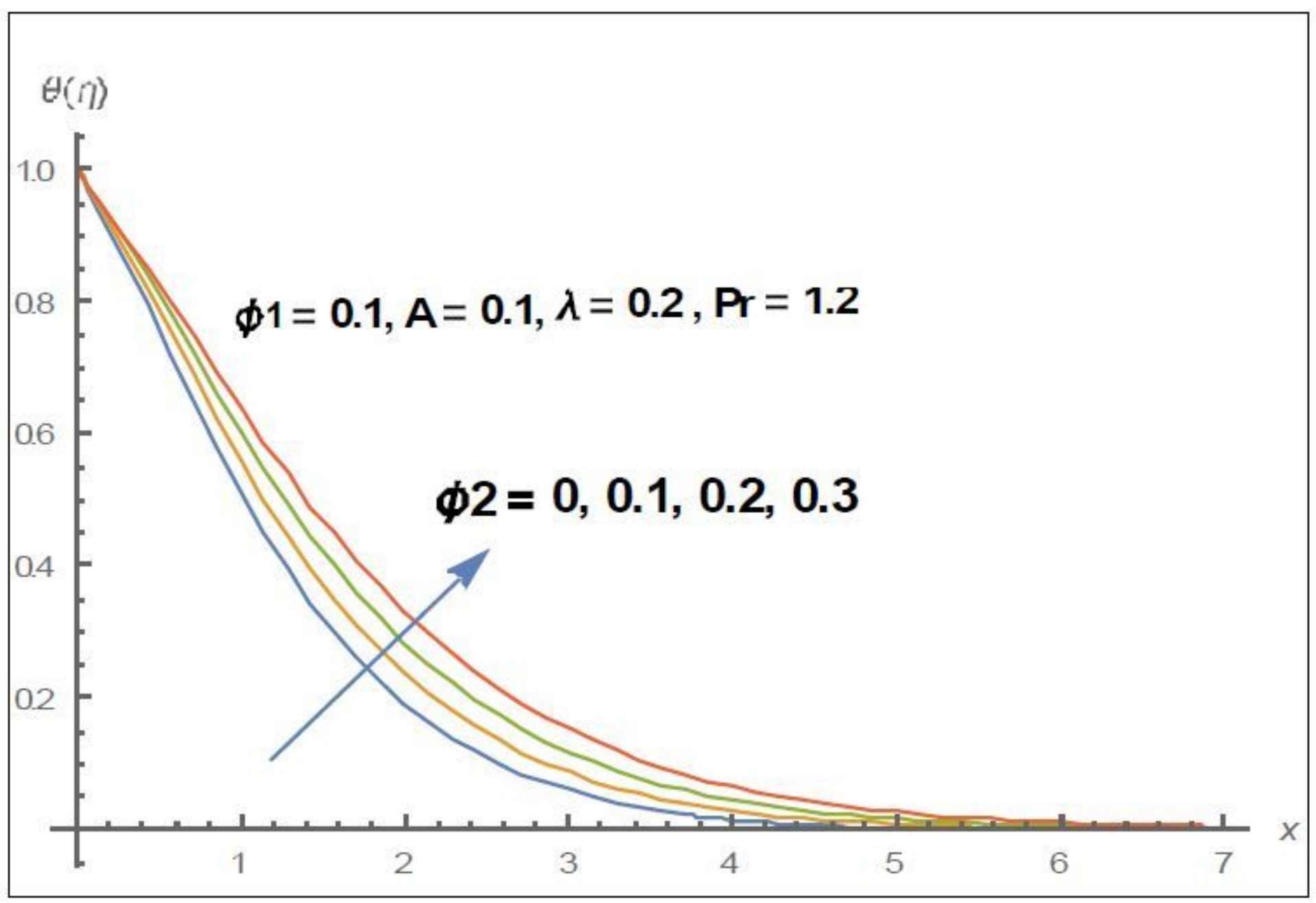

Figure 6

Due to technical limitations, figure captions can be found in the manuscript file. 


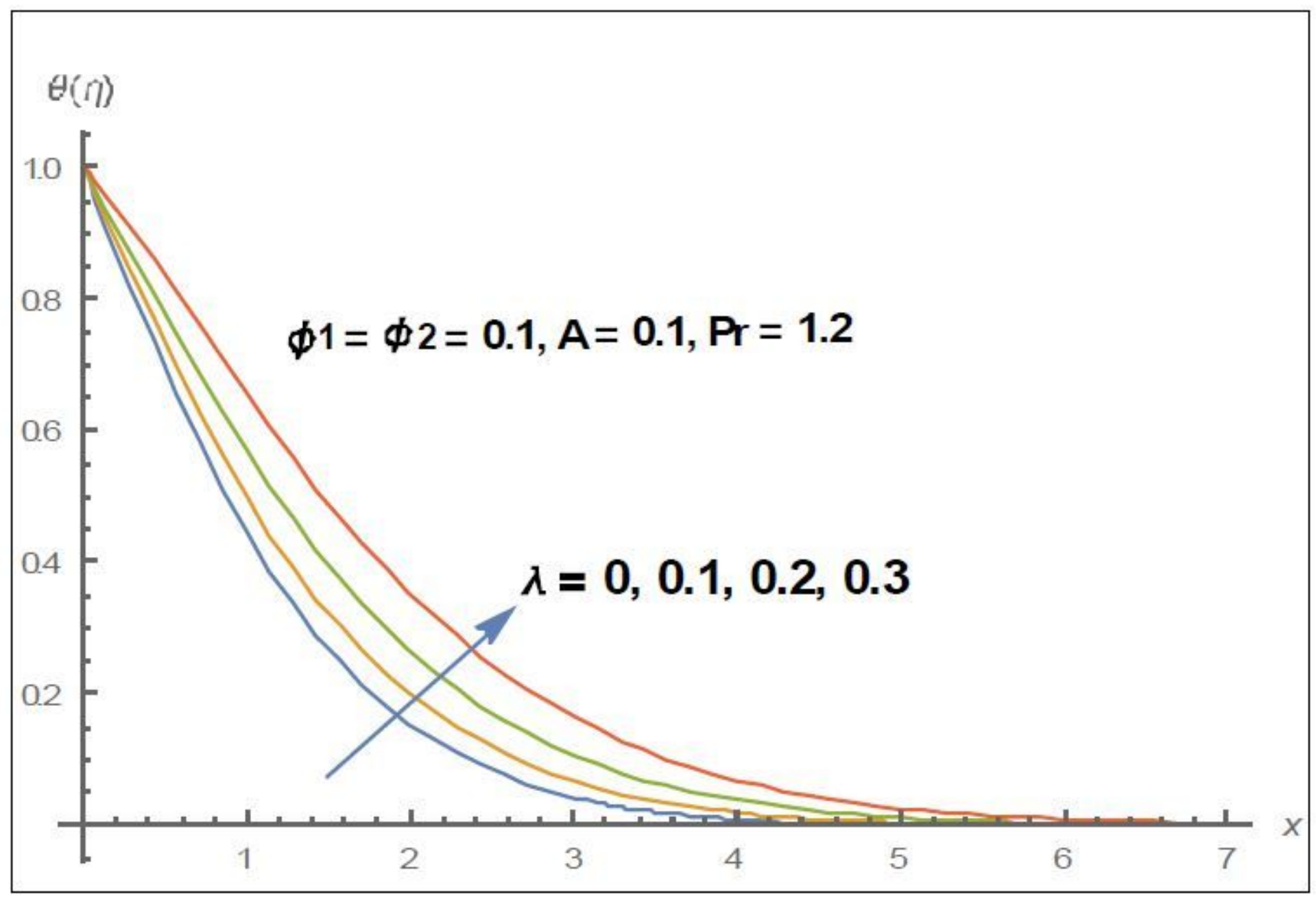

Figure 7

Due to technical limitations, figure captions can be found in the manuscript file. 


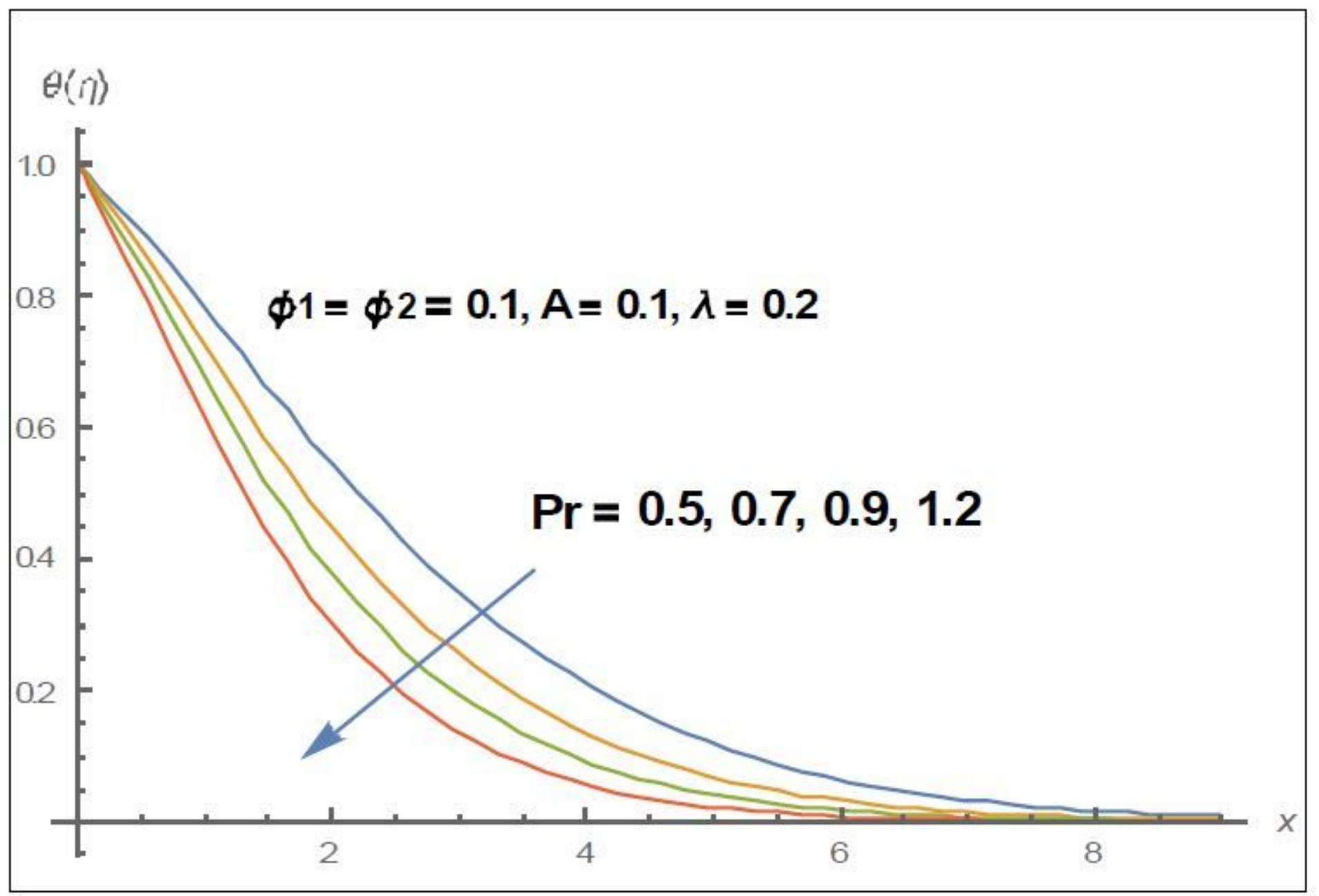

Figure 8

Due to technical limitations, figure captions can be found in the manuscript file. 


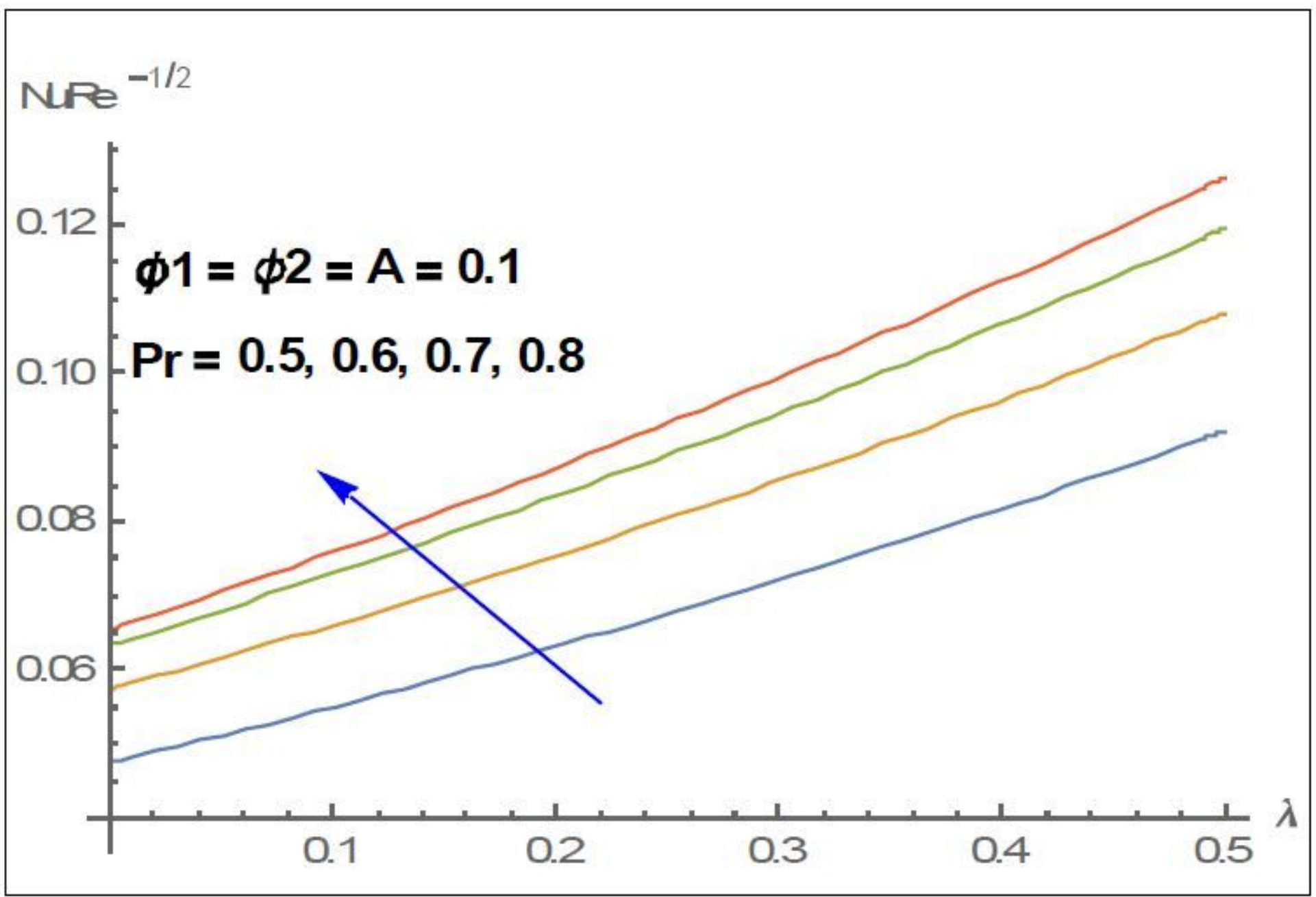

Figure 9

Due to technical limitations, figure captions can be found in the manuscript file. 


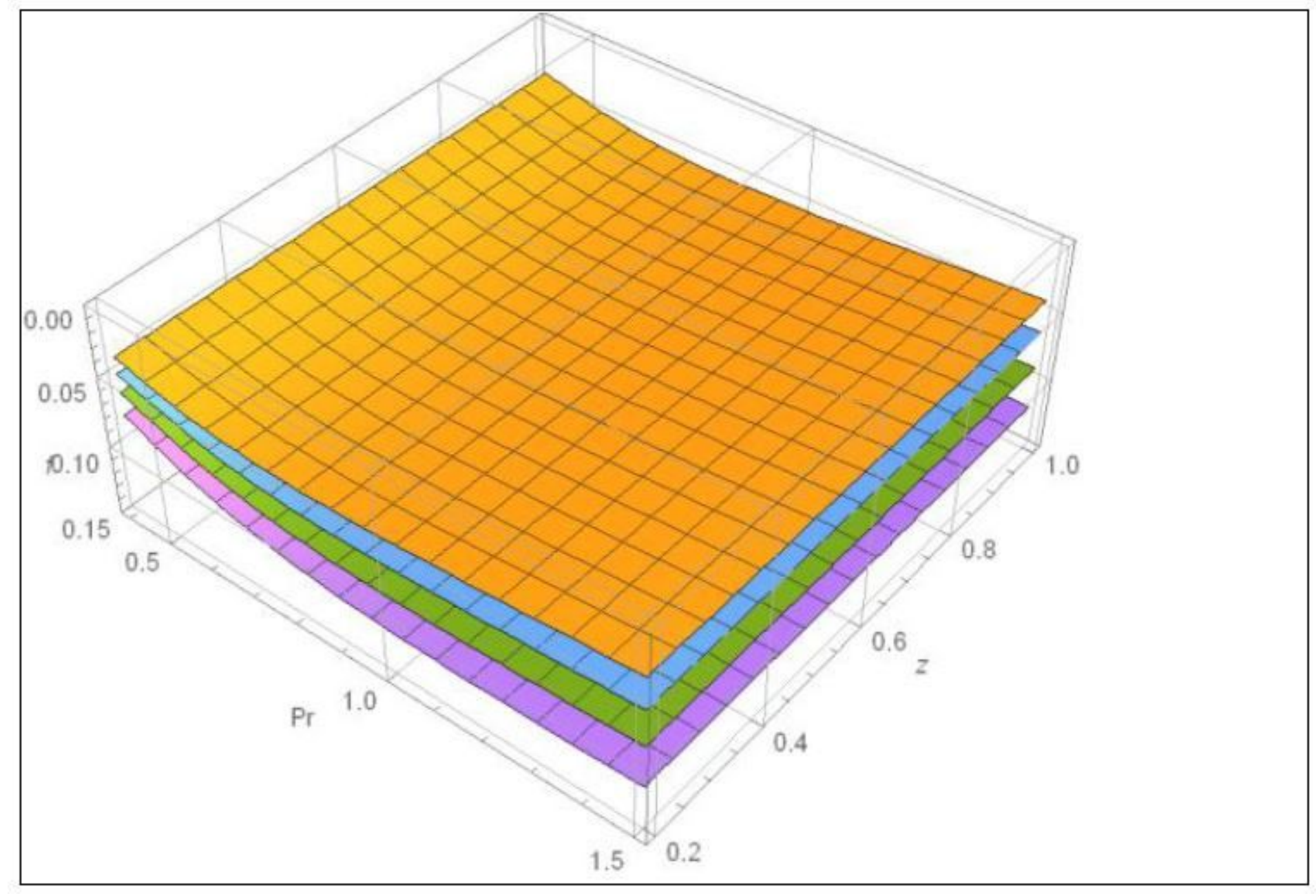

Figure 10

Due to technical limitations, figure captions can be found in the manuscript file. 


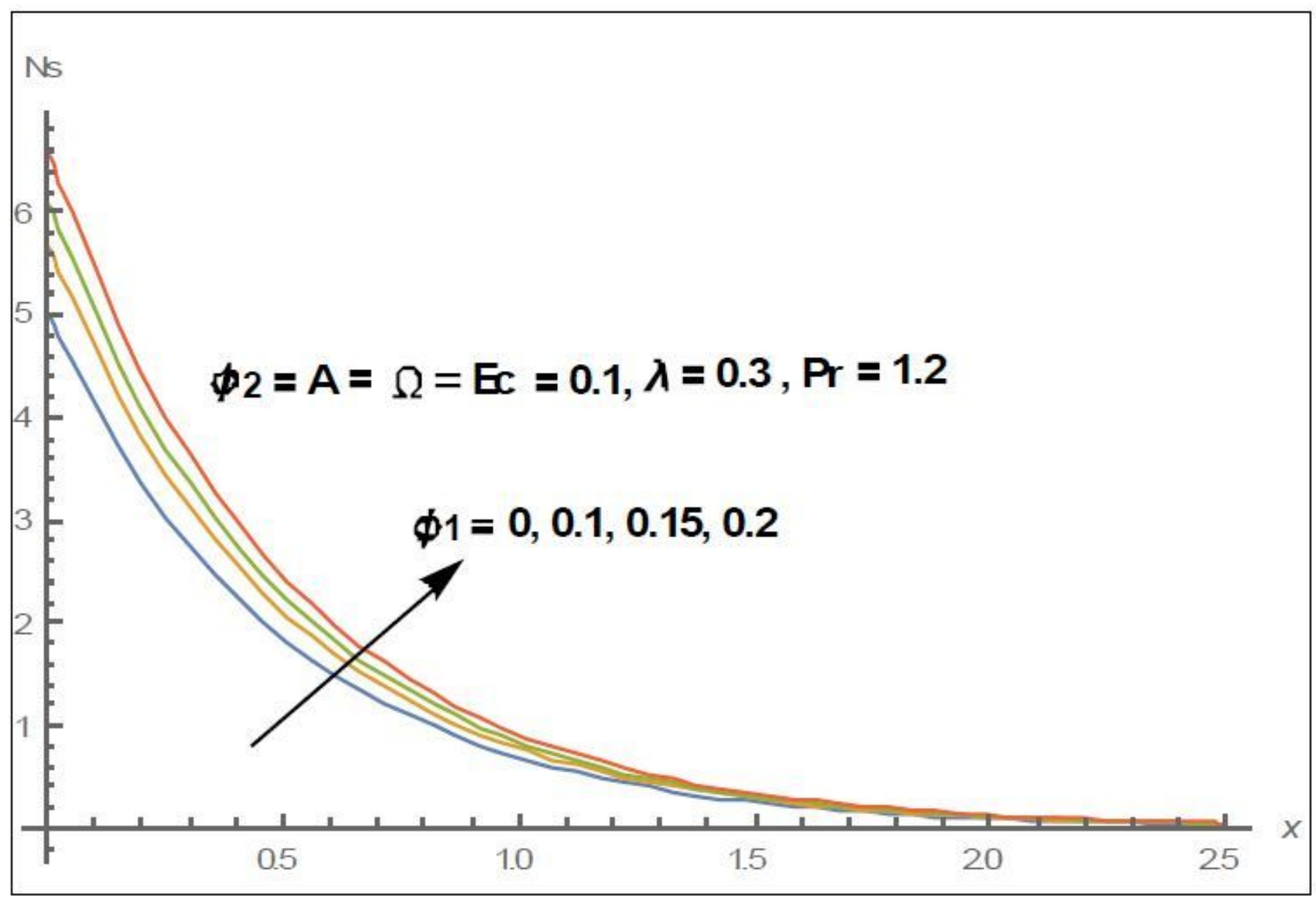

Figure 11

Due to technical limitations, figure captions can be found in the manuscript file. 


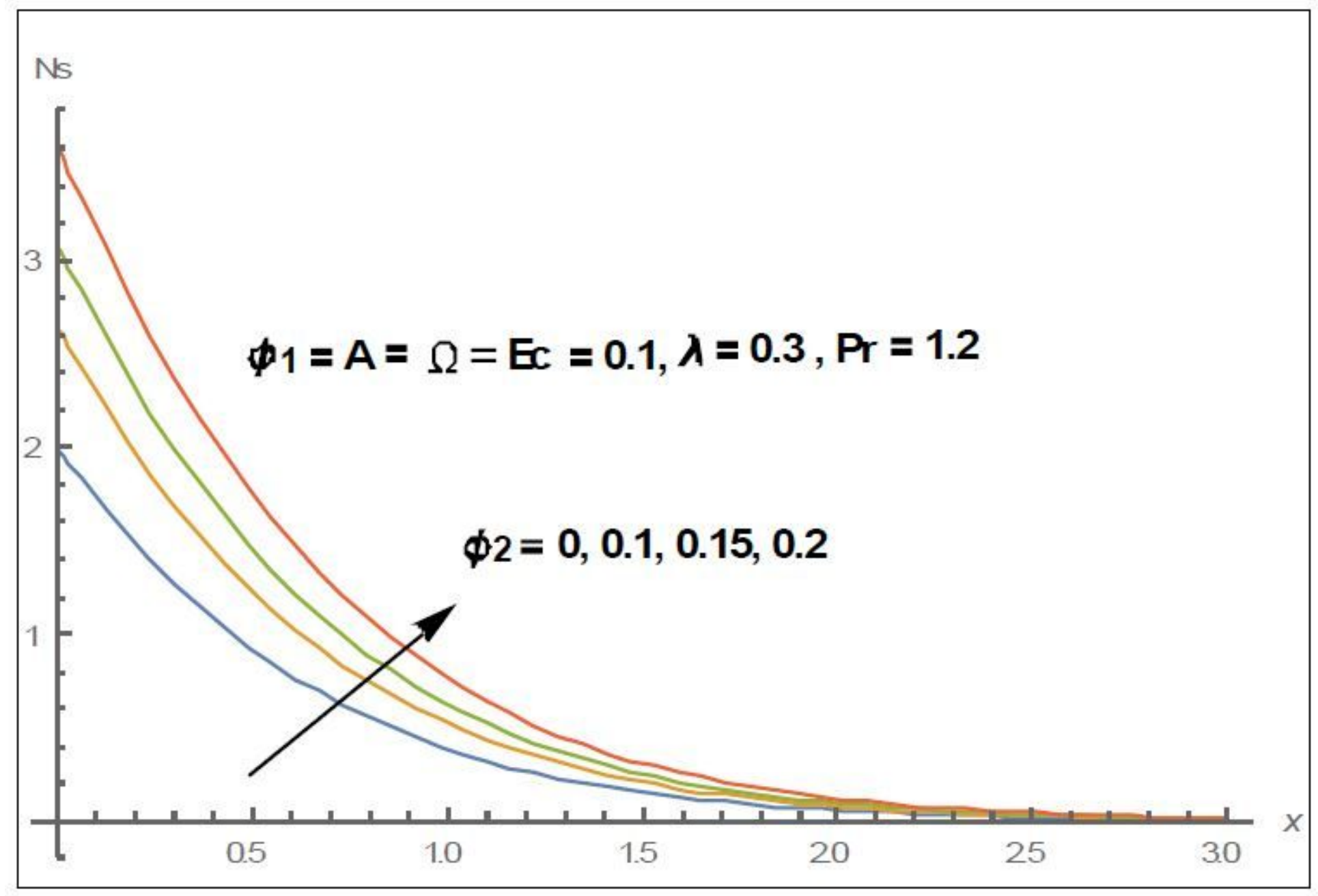

Figure 12

Due to technical limitations, figure captions can be found in the manuscript file. 


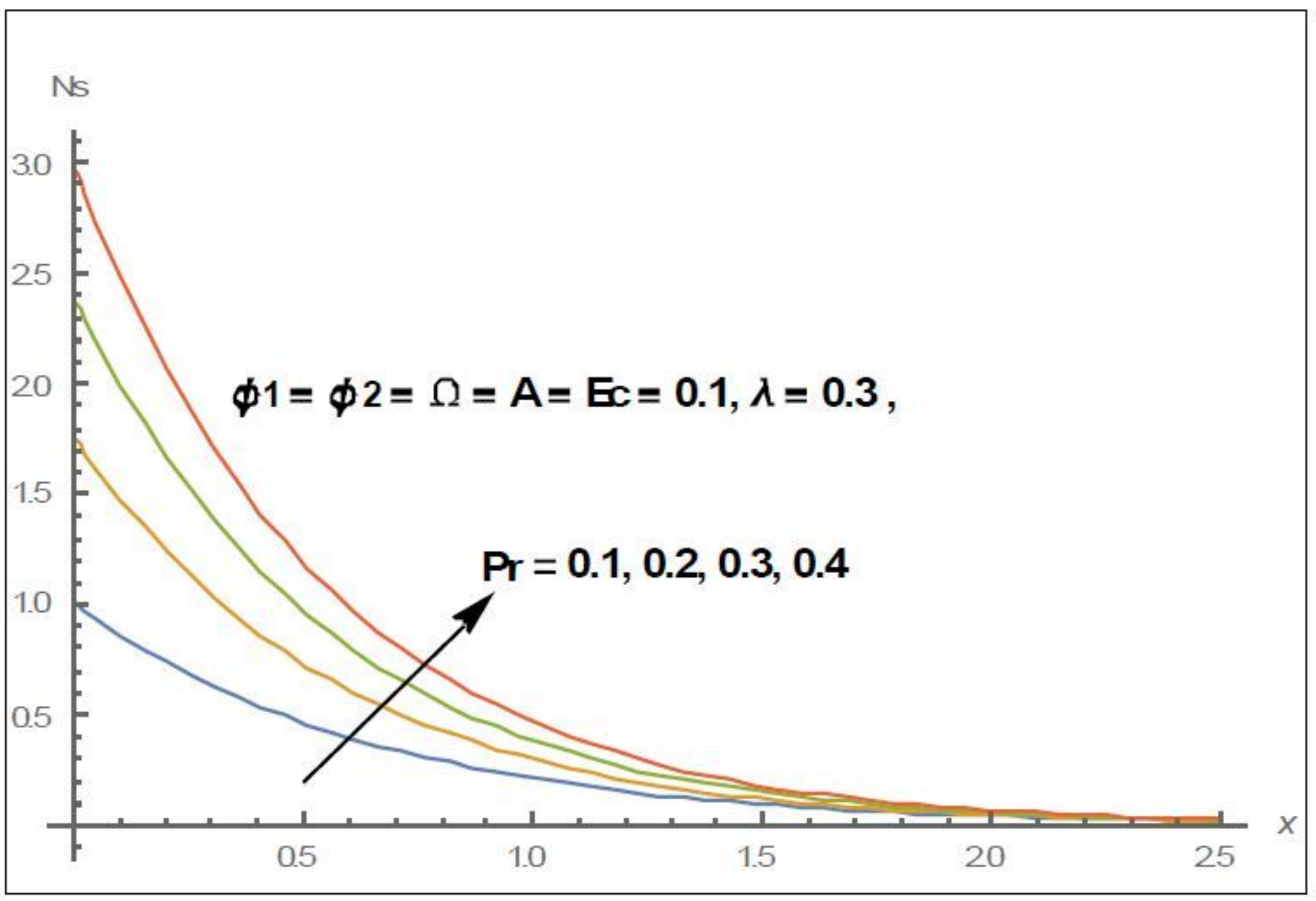

Figure 13

Due to technical limitations, figure captions can be found in the manuscript file. 


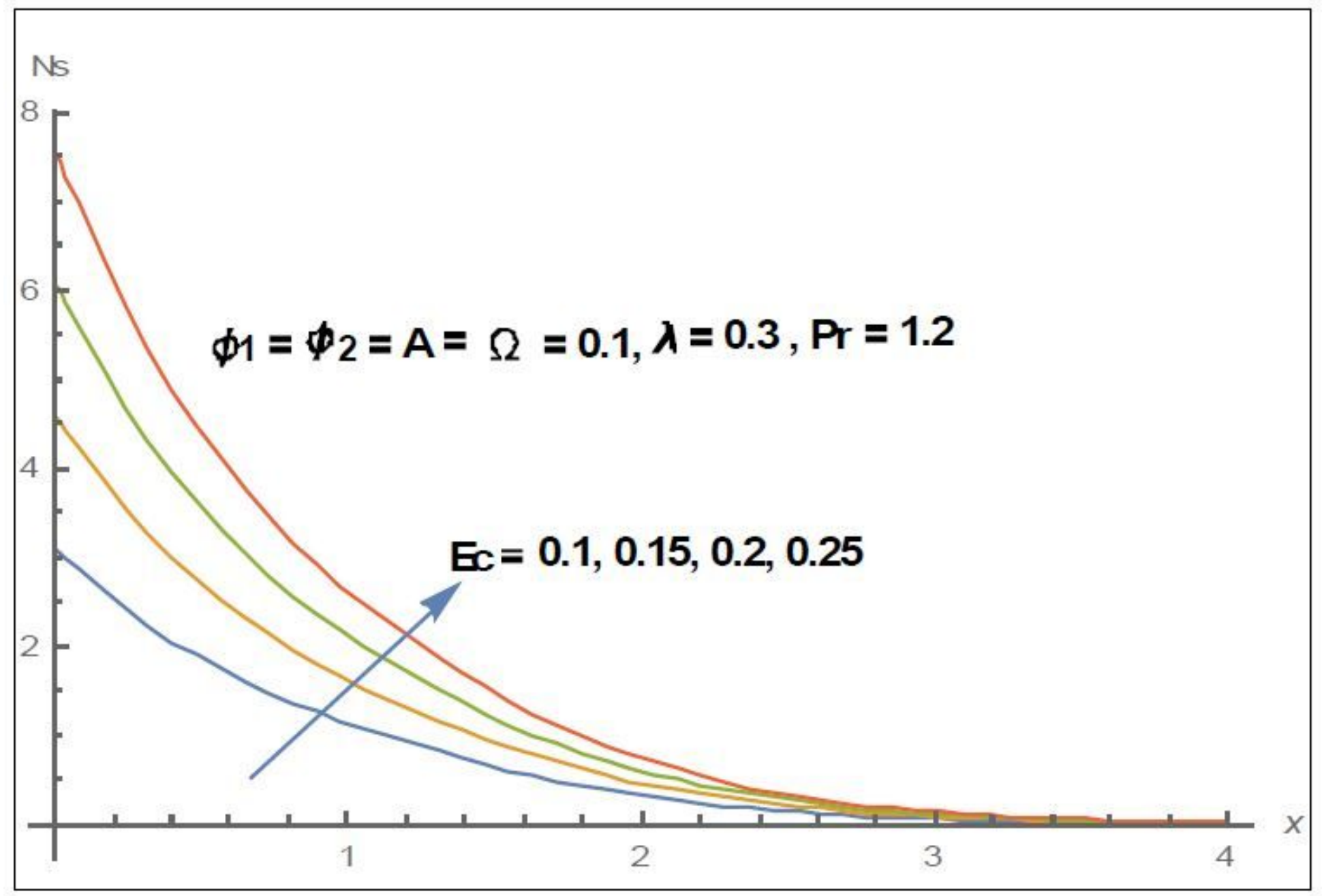

Figure 14

Due to technical limitations, figure captions can be found in the manuscript file. 


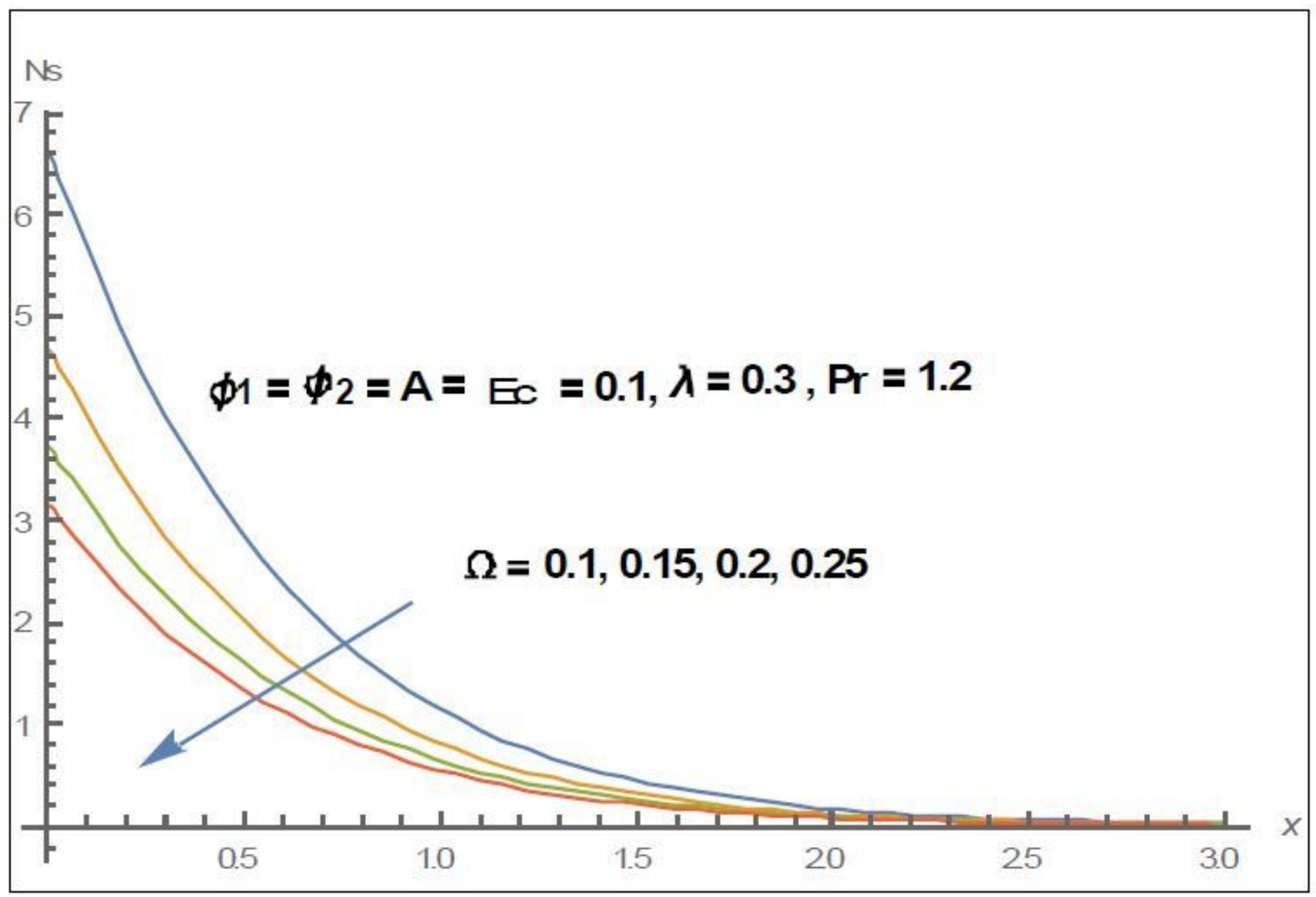

Figure 15

Due to technical limitations, figure captions can be found in the manuscript file. 